\title{
Article \\ LPS Administration Impacts Glial Immune Programs by Alternative Splicing
}

\author{
Vladimir N. Babenko*(D), Galina T. Shishkina*(D), Dmitriy A. Lanshakov (D), Ekaterina V. Sukhareva \\ and Nikolay N. Dygalo
}

check for updates

Citation: Babenko, V.N.; Shishkina, G.T.; Lanshakov, D.A.; Sukhareva, E.V.; Dygalo, N.N. LPS Administration Impacts Glial Immune Programs by Alternative Splicing. Biomolecules 2022, 12, 277. https://doi.org/10.3390/ biom12020277

Academic Editors: Camile S. Farah and Antonio Celentano

Received: 4 January 2022

Accepted: 1 February 2022

Published: 8 February 2022

Publisher's Note: MDPI stays neutral with regard to jurisdictional claims in published maps and institutional affiliations.

Copyright: (c) 2022 by the authors. Licensee MDPI, Basel, Switzerland. This article is an open access article distributed under the terms and conditions of the Creative Commons Attribution (CC BY) license (https:// creativecommons.org/licenses/by/ $4.0 /)$.

\author{
Laboratory of Functional Neurogenomics, Federal Research Center Institute of Cytology and Genetics, \\ Siberian Branch of the Russian Academy of Science, 630090 Novosibirsk, Russia; \\ lanshakov@bionet.nsc.ru (D.A.L.); sev@bionet.nsc.ru (E.V.S.); dygalo@bionet.nsc.ru (N.N.D.) \\ * Correspondence: bob@bionet.nsc.ru (V.N.B.); gtshi@bionet.nsc.ru (G.T.S.)
}

\begin{abstract}
We performed transcriptome analysis in the hippocampus $24 \mathrm{~h}$ after lipopolysaccharide (LPS) administration. We observed glial-specific genes, comprised of two-thirds of all differentially expressed genes (DEGs). We found microglial DEGs that were the most numerous in LPS group. On the contrary, differential alternative splicing (DAS) analysis revealed the most numerous DAS events in astrocytes. Besides, we observed distinct major isoform switching in the Ptbp1 gene, with skipping of exon 8 in LPS group. Ptbp 1 usually considered a pluripotency sustaining agent in brain embryonic development, according to the previous studies. Analyzing the splicing tune-up upon LPS exposure, we came to a supposition that the short Ptbp1 isoform de-represses immune-specific response by Ptbp1 adjusted splicing architecture. Additionally, the Ptbp3 (NOD1) immune-specific splicing factor has apparently been de-repressed by the Ptbp 1 short isoform in glial cells. Notably, both the Ptbp 1 and Ptbp3 genes express primarily in microglial/endothelial brain cells. We also report immune-related genes, altering their major isoforms upon LPS exposure. The results revealed immune modulating role of alternative splicing in brain.
\end{abstract}

Keywords: lipopolysaccharide; hippocampus; RNA-Seq; alternative splicing; immune response

\section{Introduction}

An increasing interest in the study of neuroinflammation is explained by its association with different neurodegenerative diseases. Inflammatory markers were detected in the rat brain even 2 years after the insult that can induce the neuropathology of Alzheimer's disease type [1]. In order to understand the molecular changes that contribute to the development of these diseases, lipopolysaccharide (LPS), the component of the outer membrane of Gram-negative bacteria, is widely used for induction of the central inflammatory process [2]. Injections of LPS also resulted in gene expression changes in brain structures, including the hippocampus [3], brain region that was implicated in the development of neurodegenerative-related psychopathology. Inflammatory- and apoptosis-related genes were revealed among these genes.

Alternative splicing manifests the flexible and rapidly evolving mechanism [4,5] of expanding the proteome diversity. The diversity is particularly vivid in the nervous system [5]. Neuroinflammation related CD44 alternative splicing was shown to affect the response of the hippocampus in the Alzheimer disease [6]. The synaptic proteins of Nrxn1-3 were shown in response to neuroinflammation by altering their isoforms ratio [7].

Along with the mature neurons, there are stage specific splicing events featuring embryonic brain development. In particular, the previous studies have identified splicing factor Ptbp 1 as one of the major factors affecting the transition from neural stem cells (NSC) to neural progenitor cells (NPS) in the course of brain-specific embryogenesis [8-10].

Recently, the study reported Ptbp1 mediating inflammatory secretome and tumorigenic processes by altering splicing landscape in senescence cells [11,12] and tumors [13], 
exhibiting Ptbp 1 as an immune repression factor. Recent study featured sophisticated interplay of Ptbp1, Ptbp2, RbFox2, and SON (SON DNA and RNA binding protein) promoting glioblastoma multiforme (GBM) genesis [14].

We addressed the impact of DAS genes in our study, assuming the major players of LPS response are astrocytes, microglia, and endothelial cells, along with consequent neuron adaptation. We analyzed the overall dynamics accounting for Ptbp 1 alteration and found Ptbp1 exon 8 skipping may modulate immune competent response, primarily in microglial, astrocyte and endothelial cells in hippocampus.

\section{Materials and Methods}

\subsection{Animals}

Adult male Wistar rats (2.5 months of age) were used in the experiments. Animals were housed individually in polycarbonate cages $(27.7 \times 44 \times 15 \mathrm{~cm}=\mathrm{w} \times 1 \times \mathrm{h})$ with free access to food and water.

All animal use procedures were supervised and specifically approved by the ethic committee of the Institute of Cytology and Genetics, in accordance with the guidelines of the Ministry of Public Health of Russia (supplement to order N 267 of 19 June 2003) and European Council Directive (86/609/EEC). The middle cerebral artery occlusion and LPS administration into striatum included all measures to minimize rat suffering.

\subsection{LPS Administration}

The global agenda of our research is getting an insight on the involvement of strokeinduced inflammatory activation on the hippocampus by determining the genes directly affected by pro-inflammatory stimuli. Since middle cerebral artery occlusion (MCAO) causes the most severe neuronal damage in the ipsilateral striatum in rats [15] we have chosen the striatum for central LPS administration, thus complying to the published protocol for an acute rat model of local neuroinflammation in this brain structure [16].

LPS (30 $\mu \mathrm{g}$ in $4 \mu \mathrm{L}$ of sterile saline) from Escherichia coli, serotype 055:B5 (SigmaAldrich Corp., St. Louis, MO, USA), or an appropriate volume of saline (SAL) were infused stereotactically into the right striatum under isoflurane anesthesia ( $4 \%$ isoflurane for induction, $2.5 \%$ for maintenance in $\mathrm{O} 2$ at a flow rate of $1 \mathrm{~L} / \mathrm{min}$ ). We used the following coordinates for drug infusions: $\mathrm{AP}=+0.5 \mathrm{~mm}, \mathrm{ML}=+3 \mathrm{~mm}$, and $\mathrm{DV}=-5.5 / 4.5 \mathrm{~mm}$ [16] As was shown previously, this LPS treatment regimen also effectively provoked an acute neuroinflammation in the rat hippocampus [15].

\subsection{Collecting Hippocampal Samples}

Twenty-four hours after LPS administration, the rats were sacrificed by rapid decapitation. Brains were quickly extracted and ipsilateral hippocampi $(n=3$ for each group of LPS, SAL) were rapidly isolated, and each was placed in an Eppendorf tube with $1 \mathrm{~mL}$ of buffer containing an RNase inhibitor (RNAlater) at room temperature. After that, the tube was transferred to ice, after the end of hippocampal collection, stored overnight at $+4{ }^{\circ} \mathrm{C}$, and then at $-80^{\circ} \mathrm{C}$ until the analysis of gene expression patterns.

\subsection{RNA-Sequencing and Data Analysis}

RNA-seq was performed in JSC Genoanalytica (Moscow, Russia; Available online: http:/ / genoanalytica.ru, accessed on 15 November 2021). For this, total RNA was extracted from the ipsilateral hippocampus in the ischemic and LPS-infused rats with Trisol reagent, according to manufacture instruction. Quality was checked with the BioAnalyser and RNA 6000 Nano kit (Agilent, Santa Clara, CA, USA). PolyA RNA was purified with Dynabeads ${ }^{\circledR}$ mRNA Purification kit (Ambion, Austin, TX, USA). Illumina library was made from polyA NEBNext ${ }^{\circledR}$ Ultra $^{\text {TM }}$ II RNA Library Prep (NEB, Ipswich, MA, USA), according to manual. Sequencing was performed on HiSeq1500, with $50 \mathrm{bp}$ read length. At least 30 million of reads were generated for each sample. 
The raw reads from RNA-seq experiments were trimmed for quality (phred $\geq 20$ ) and length (bp $\geq 32$ ) using the Trimmomatic v. 3.2.2 [17]. Reads were mapped to the Rnor_6.0 genome with STAR aligner [18] and differentially expressed transcripts were inferred by Cuffdiff software v.2.1.1, accessed on 10 May 2021, http://cole-trapnell-lab.github.io/ cufflinks / manual/ [19]. Genes with an adjusted p value (padj) less than 0.05 were classified as significantly differentially expressed genes (DEGs). A total of 34,600 transcripts were in the reference (annotation) set.

Differential alternative splicing (DAS) analysis has been performed using rMATs software, version 4.2.2., accessed on 10 May 2021 http: / / rnaseq-mats.sourceforge.net/ download.html [20]. Only exon skipping (ES) events were considered.

\subsection{Statistical Methods}

GO enrichment analysis has been performed, with the string-db.org routine (stringdb.org; accessed 15 December 2021). GO enrichment non-redundant grouping was performed with GOMCL software [21], accessed on 10 October 2021; https://github.com/ Guannan-Wang/GOMCL).

Principal component analysis (PCA) has been performed by commercial XLSTAT software (accessed on 10 May 2021; https:/ / xlstat.com). As a distance matrix, a Pearson pairwise correlation matrix of DEGs expression profiles was used. Agglomerative hierarchical clustering (AHC) was employed using XLSTAT software. The AHC parameter set was: (similarity: Pearson correlation coefficient; agglomeration method: unweighted pair-group average; center: no; reduce: no; truncation: automatic-inertia). Heatmap construction was performed with a self-organizing map (SOM) algorithm employing XLSTAT software.

\section{Results}

\subsection{GO DEGs Analysis}

Due to the brain invasion and consequent post-operational inflammation in both samples (SAL and LPS), we filtered the DEGs sample for the genes maintaining abs $(\log 2$ fold $)>2$. This resulted in final DEGs set of 87 entries (Table S1, Supplementary Materials).

Analysis of the gene ontology (GO) in the biological process yielded 284 entries (Table S2, Supplementary Materials). To obtain the core DEGs set, we applied a GO clustering algorithm GOMCL (Wang et al., 2020), which yielded just one GO supercluster consisting of 37 distinct DEGs, encompassing the 284-fold initial GO BP annotation list (Table S1, Supplementary Materials). This way we were able to see the non-redundant DEGs network, represented in Figure 1.

We employed PCA analysis (Figure 2a) outlining three clusters depicted in Figure 1 to proceed on details of each cluster. We found that Hcrt, attenuated in the LPS group, refers to hormone activity (Figure 2b), while two other clusters manifest inherent distinct coordinated responses to LPS exposure (Figure 2c,d). Elucidating glial-specific genes in the cluster using brain cells expression atlas, presented in [22] we were able to relate 24 genes as glial-specific ones (Figure 2e,f).

We found Shox2 DEG, induced by an outlier value in the SAL1 sample, equaling 7.4 FPKM, while five other samples maintaining it as less that 0.07 (avg $=0.05$; stddev $=0.03)$, so we discarded it.

Overall, we came to 36 DEGs as the core DEGs spanning the GO spectra. Microglialspecific genes were prevalent (22 DEGs) followed by endothelial genes (Fos, Sele; Figure 2e) and astrocytes display 2 genes (Serping1, Fcnb). Notably, Fos DEG expresses virtually in all cell types, most prominently in astrocytes [22] implying the assignment of this gene to epithelial cells may be due to Sele DEG co-variation. Other DEGs were not immediately assigned to glial cells; still, more than half of DEGs (24 entries) were assigned to be glial rather confidently. 


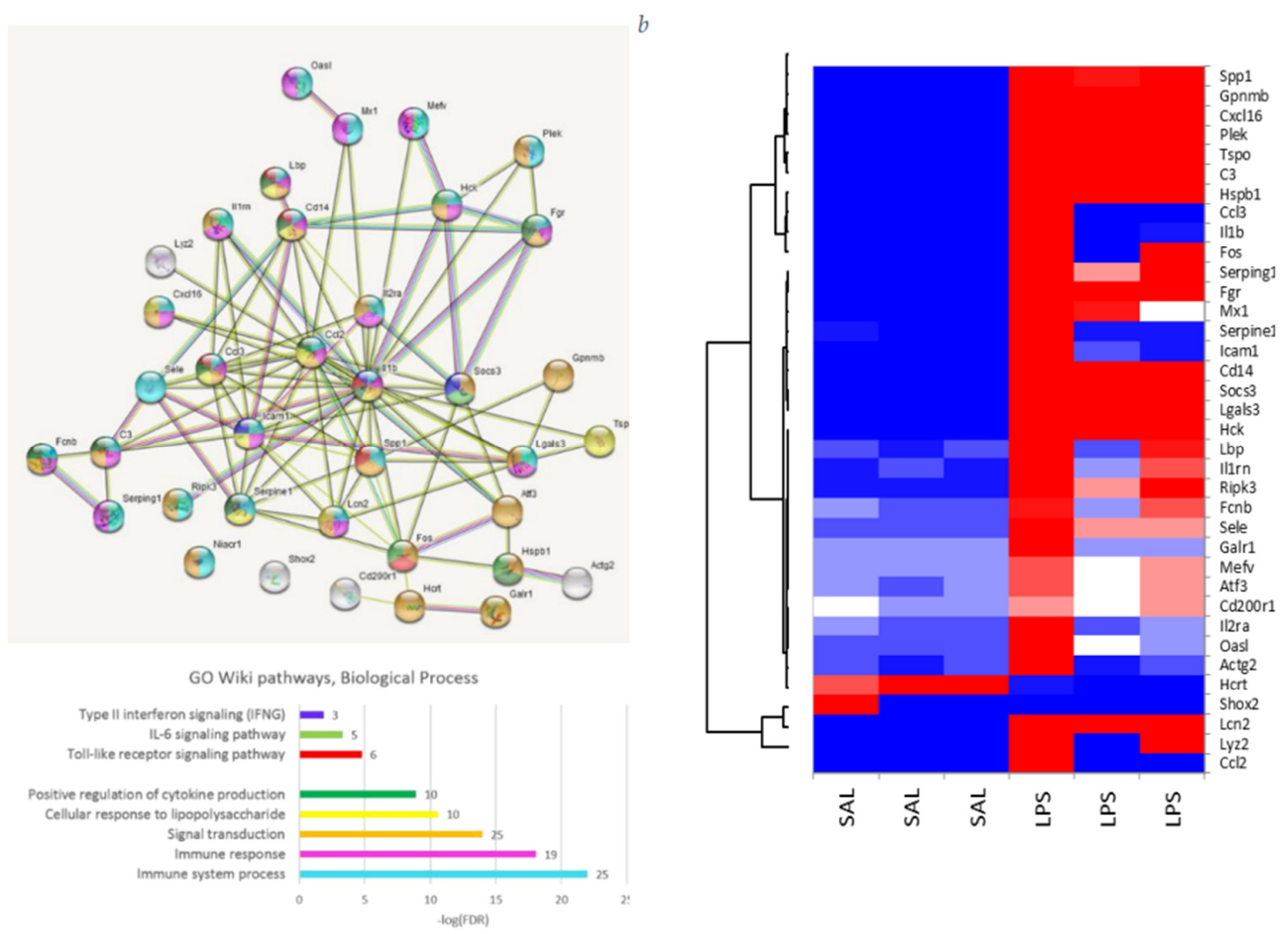

Figure 1. (a) GO annotation, 37 core DEGs in SAL vs. LPS comparison. Selected are several relevant GO biological process and wiki pathways from Table S1. Colors on the plot correspond to those on the figure. Number of DEGs per GO term attached as bar labels. (b) Heatmap of 37 DEGs, clustering underlines three clusters: small one (Hcrt and Shox2), attenuated in LPS group, and two others (specific to the LPS group).

\subsection{DAS Genes Analysis}

We identified 49 differentially, alternatively spliced genes with ES event alteration FDR $<0.05$ between SAL and LPS groups, presented in Table S3, Supplementary Materials. Only one DAS event per gene was observed. While no significantly enriched GO terms were featured for the set, we ascribed them manually.

We observed 3 ncRNA and 46 coding RNAs, one currently not annotated in public repositories (AABR07039316.1) and overlapping two small coding genes. Manual annotation elucidated several functional categories: lipid and lipolysis related (three DAS genes: Lsr, Irf3, and Lrp8); splicing factors and chromatin rearrangement (Ptbp1, Rnps1, and Rbm34), mitosis associated centromere proteins (Cep126 and Cep295), coagulation associated factors $(F 8, v W a 5 b 2)$, and glycoproteins/development related one (Eogt; Notch3-signaling), AABR07019088.1 (AS RNA of Nduf3b).

A total of 49 genes, featuring DAS ES events, maintain 9 median isoforms per gene (Table S4, Supplementary Materials), as annotated in the NCBI repository. We observed 3 median isoforms per gene for the same 49 DAS genes in our RNA-seq data, when including all ES events detected. The Pearson correlation of the RNA observed and expected (NCBI) numbers is quite significant (equaling $r=0.665, \mathrm{df}=45$ (excluding three 
non-annotated RNA), $p<1 \times 10^{-5}$ ) and implying we maintain good sensitivity with our AS detection algorithm.

Pursuing further analysis and keeping in mind the previous studies on neuroinflammastion, we suppose that: (a) the major role in LPS response attributed to glial cells; (b) non-random DAS ES events invoke the corresponding genes expression modulation (up or down).

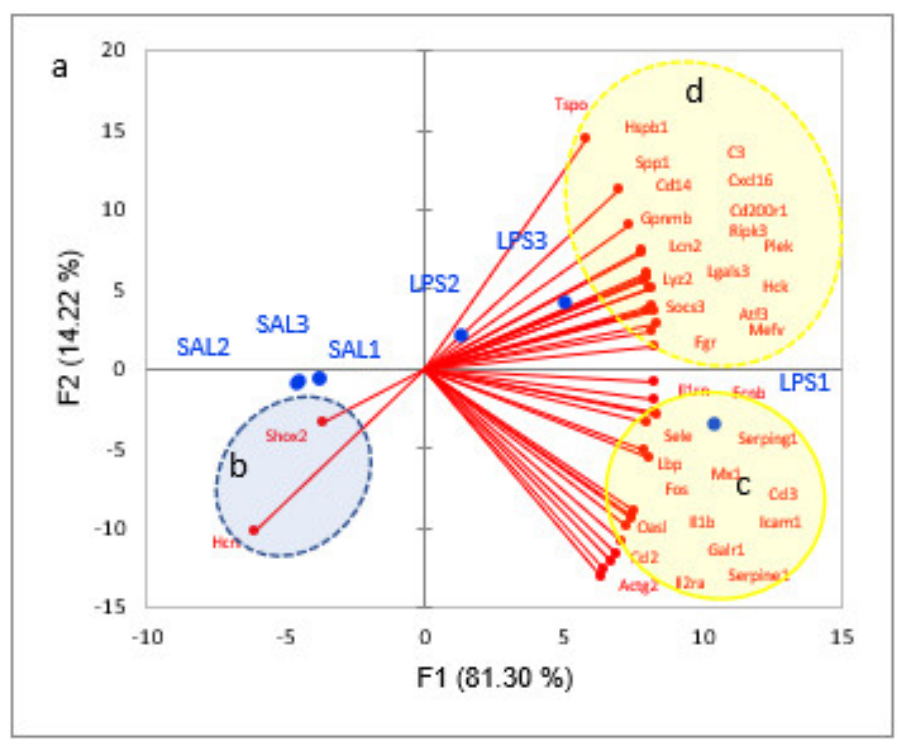

b

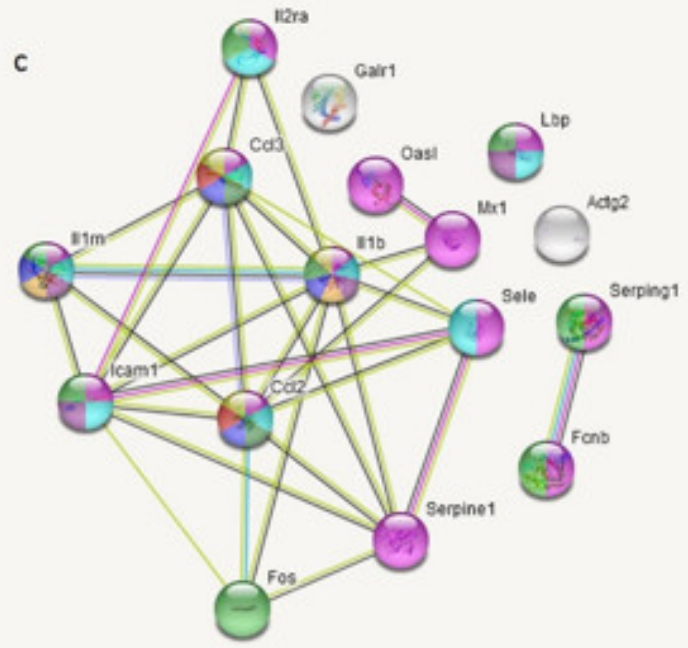

\section{$d$}
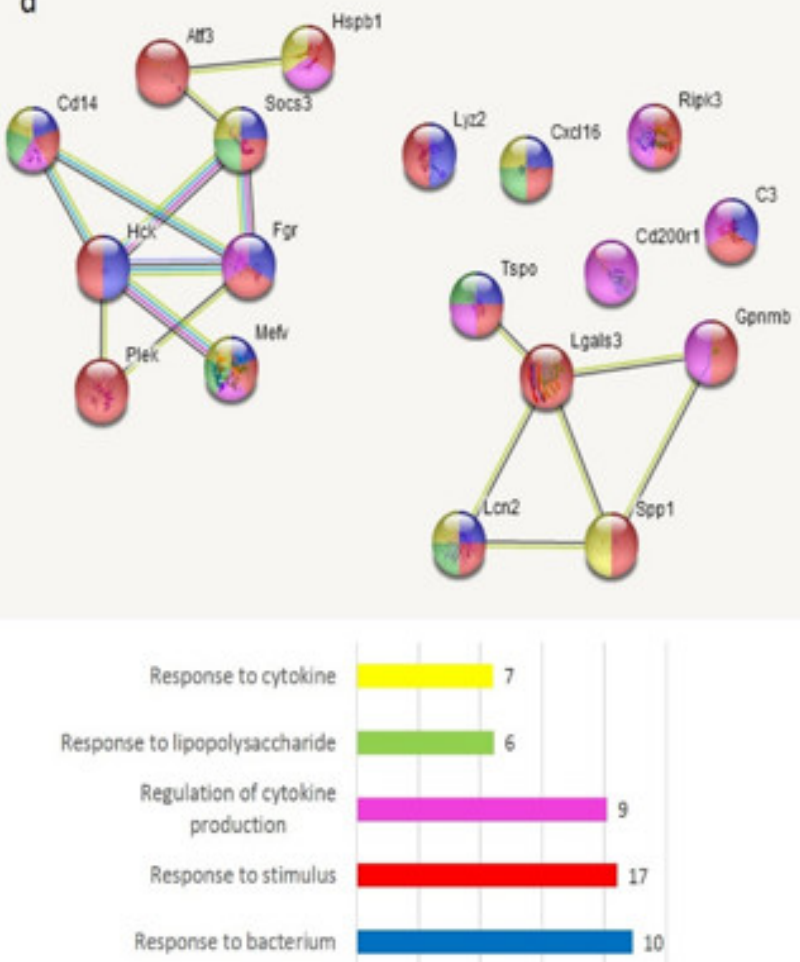

immune system process
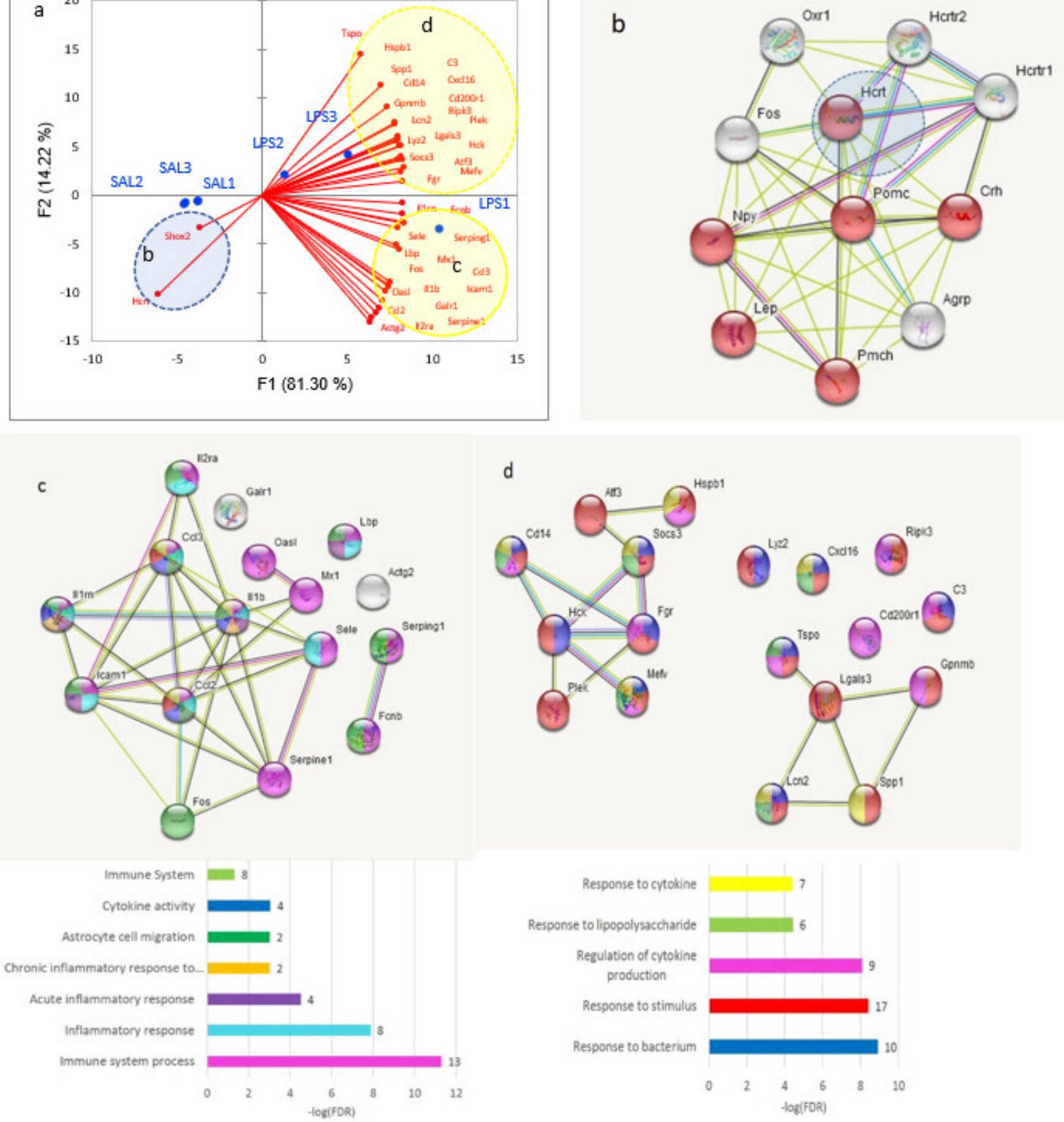

Figure 2. Cont. 

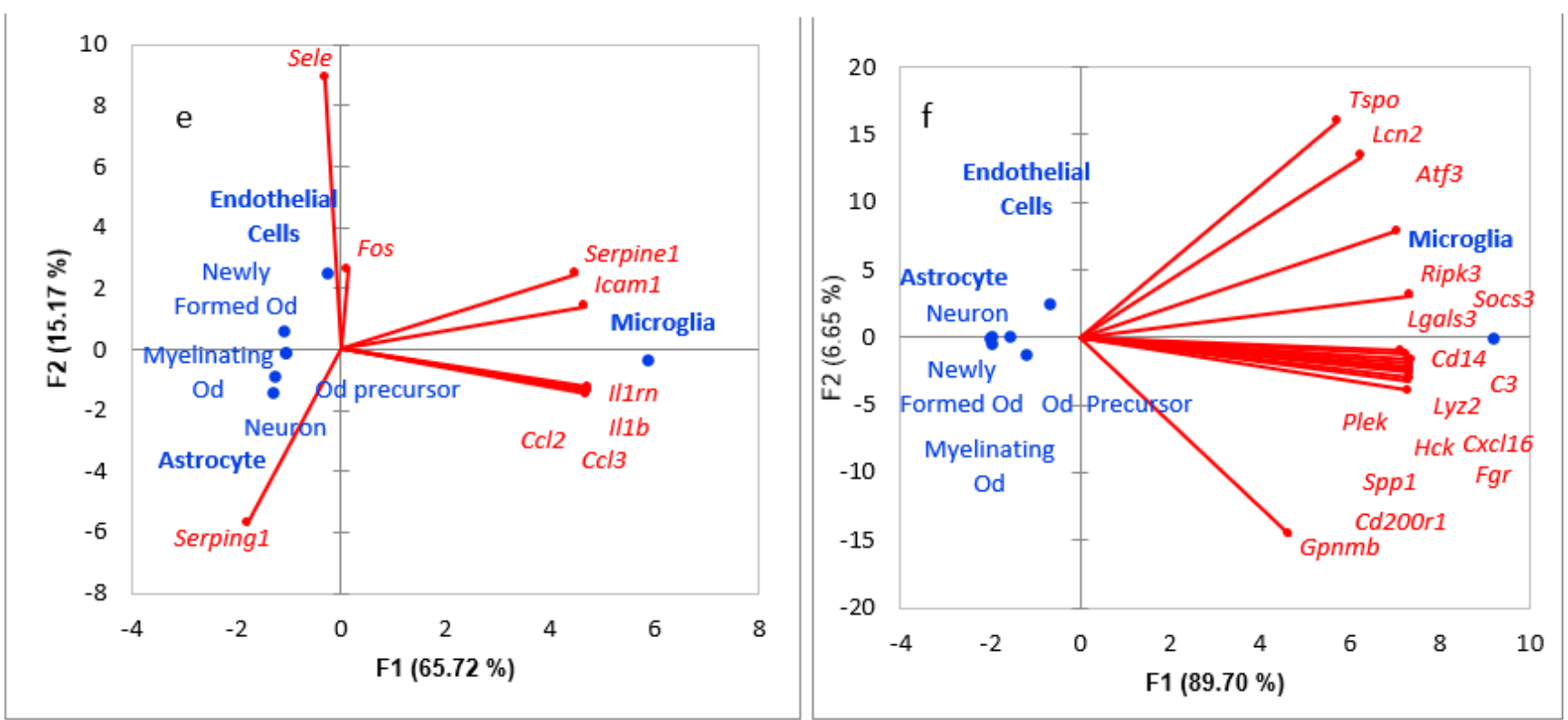

Figure 2. (a) 3 clusters (b, c, d) of 37 DEGs encircled by ovals; (b) SAL-related Hcrt environment unveils its relation to GO:0005179 (hormone activity; 6 genes of 108; FDR $<1.77 \times 10^{-8}$ ), depressed in LPS; (c) coordinated mixed glial genes featuring microglial (Serpine icam1, il1 rp, il1b, ccl2, ccl3, and Fos), endothelial (Sele and Fos), and astrocyte (Serping, Fonb, and Fos)) cells; 10 DEGs; (d) microglial coordinated cluster (16 DEGs); (e) PCA plot of (c) cluster DEGs across cell lines expression profiles [22]; (f) PCA plot of (d) cluster DEGs across cell line expression profiles. 'Od' stands for Olygodendrocyte.

\subsubsection{Cell-Specific Expression of DAS Genes}

First, we assessed the distribution of 49 DAS gene (DAS FDR $<0.05$ ) expression profiles across SAL and LPS samples (Figure 3). Upon analysis of Figure 3 we may state there is certain preference of DAS genes to SAL/LPS samples, based on their PCA of expression profiles, depicted in Figure 3. In particular, 17 glial DAS genes augmented their expression in the LPS group (Figure 3; bold typed genes, orange circles), while only 6 glial DAS genes attenuated it in LPS group (Figure 3; blue circles).

Based on [22], the brain cell-specific expression profiles we assessed showed 49 for cell specific expression (Figure 4). We identified the gene as a cell specific on when it maintains the highest expression among seven cell types. As expected, the most AS events took place in neuron specific genes (13-16 DAS genes). Still, we observed distinct glia specific genes in Figure 4 (colored gene names in the first column).

9 genes were not found in Atlas. In particular, cep126, сер295 are absent, while 21 cep genes family was found highly expressed specifically in endothelial cells (order of magnitude higher than in any other cell type), but further were omitted from the analysis due to high non-specificity to LPS issue. No noncoding (ncRNA)/unannotated ones were found in Atlas, in particular: Spaca, AABR07019088.1, AABR07039316.1, AABR07069473.1. Ogt was used for annotation of Eogt, Stk30 for Mok. Both Stk30 and Ogt are astrocyte specific genes. Thus, we maintained 42 genes as annotated for cell specificity.

\subsubsection{Sampled DAS Genes}

Based on Figure 4 we defined 3 glia specific DAS groups comprising 17 genes. To further confirm their specificity within glial cells we built PCA plot for 3 glial cells (Figure 5). Most of the DAS genes were observed in astrocytes, according to Figure 5. Overall, we compiled 24 glial-specific DAS genes presented in Table 1. 


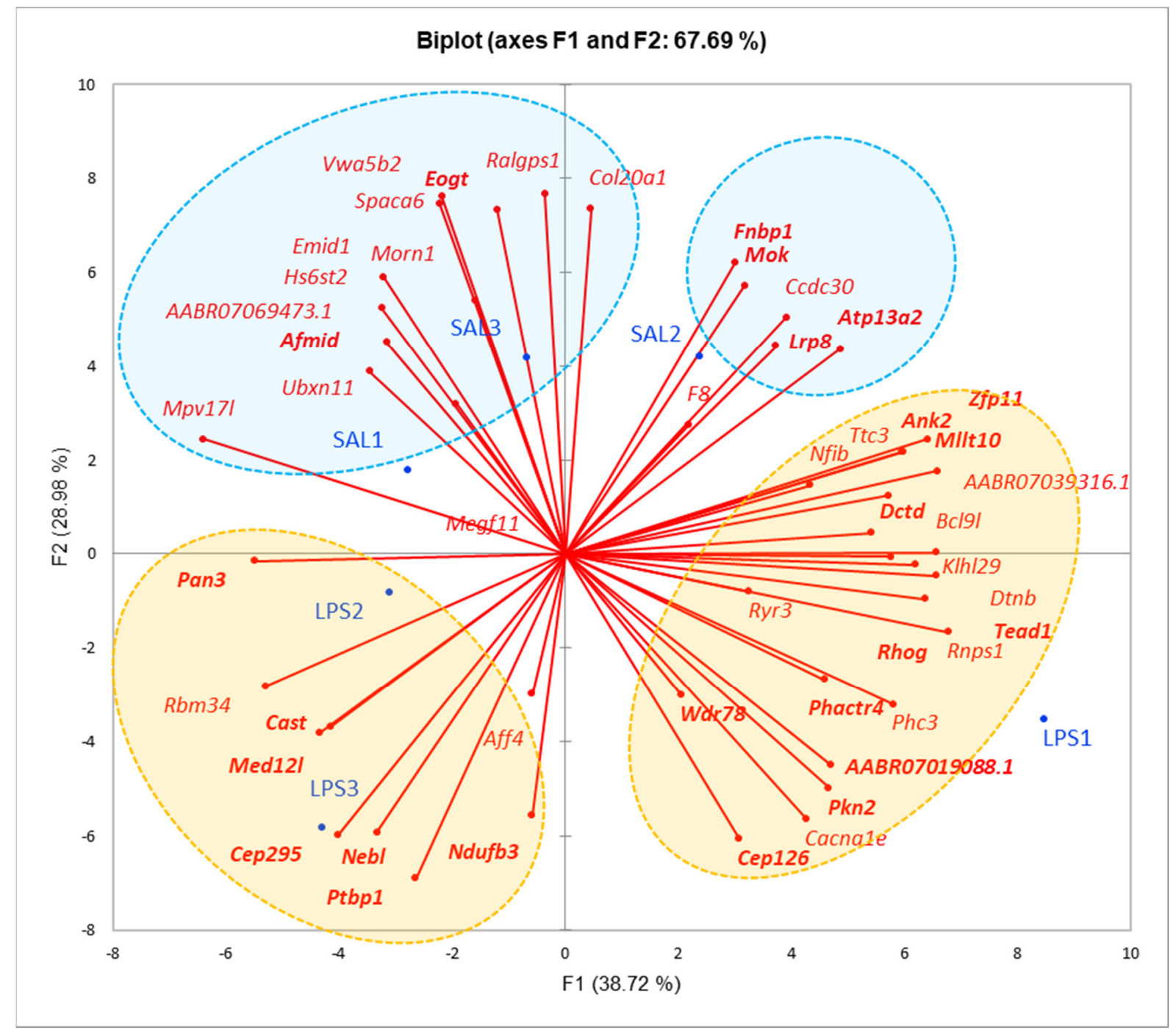

Figure 3. PCA plot, based on expression profiles of 49 DAS genes (FDR $<0.05$ ), across 6 samples, manifest certain preference to the samples. Orange shaded are DAS genes with enhanced expression in LPS, blue shaded are DAS genes attenuated their expression in LPS. Bold typed are glia-related genes (see text below).

Table 1. The list of 24 DAS genes preference in glial cells. Top significant DAS genes are bold typed.

\begin{tabular}{ccc}
\hline Endothelial Cells & Microglia & Astrocytes \\
\hline Ptbp1 & Ptbp1 & Ank2 \\
Cast & Rhog & Med12l \\
Phactr4 & Atp13a2 & Tead1 \\
Nebl & Afmid & Pan3 \\
Lrp8 & & Milt10 \\
Pkn2 & Ptn2 \\
Ndufb3 & Fnbp1 \\
& & Zfp1 \\
& & Wrd78 \\
& & Eogt \\
& & Dctd \\
& & Phc3 \\
& & Mok \\
\hline
\end{tabular}




\begin{tabular}{|c|c|c|c|c|c|c|c|c|}
\hline 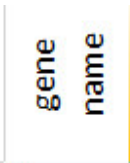 & 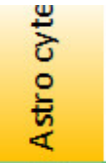 & $\begin{array}{l}\frac{c}{0} \\
\frac{0}{2} \\
\frac{d}{2}\end{array}$ & ๖் & 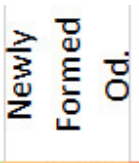 & 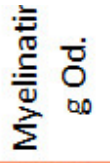 & $\begin{array}{l}\frac{\pi}{00} \\
\frac{0}{0} \\
\frac{0}{\Sigma}\end{array}$ & $\begin{array}{l}\text { 誉 } \\
\text { 吾 } \\
\text { 음 } \\
\text { 네 }\end{array}$ & 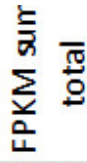 \\
\hline Rhog & 9.9 & 2.2 & 20.6 & 105.8 & 211.5 & 315.7 & 30.6 & 696.3 \\
\hline Ptbp1 & 54.2 & 20.3 & 28.4 & 26.7 & 6.9 & 127.1 & 104.2 & 367.8 \\
\hline Ndufb3 & 21.8 & 52.5 & 46.6 & 42.5 & 43.3 & 25.5 & 59.9 & 292.1 \\
\hline Atp13a2 & 14.1 & 67.5 & 27 & 21.2 & 16.9 & 61.8 & 11 & 219.5 \\
\hline Ttc3 & 35.7 & 81.9 & 49.1 & 18.4 & 5.6 & 3.1 & 15.2 & 209 \\
\hline Fnbp1 & 50.7 & 15.5 & 6 & 34.2 & 75.2 & 10.7 & 2.3 & 194.6 \\
\hline Emid1 & 28.4 & 5.5 & 122.2 & 26.1 & 0.6 & 0.4 & 1.2 & 184.4 \\
\hline Aff4 & 22.1 & 34.8 & 18.2 & 15.2 & 4.4 & 5.9 & 21.4 & 122 \\
\hline Ank2 & 54.6 & 28.4 & 13.6 & 10.9 & 9.1 & 1.5 & 0.4 & 118.5 \\
\hline Lrp8 & 12.3 & 26.2 & 5.9 & 1.4 & 0.2 & 0.4 & 58.5 & 104.9 \\
\hline Nfib & 28.9 & 43.1 & 13.6 & 4.3 & 1.6 & 0.2 & 11.4 & 103.1 \\
\hline Pan3 & 19.3 & 15 & 11.1 & 6.9 & 1.6 & 9.4 & 20.8 & 84.1 \\
\hline Megf11 & 0.3 & 2.5 & 44.7 & 11.9 & 0.3 & 0.2 & 0.2 & 60.1 \\
\hline Mllt10 & 14.6 & 6.7 & 6.4 & 4.7 & 1.5 & 3.1 & 13 & 50 \\
\hline Pkn2 & 10.6 & 4.5 & 8.1 & 4.5 & 1.3 & 1.4 & 17.4 & 47.8 \\
\hline Dtnb & 8.3 & 9.5 & 13.8 & 6.1 & 2.2 & 3.1 & 4.5 & 47.5 \\
\hline Bcl9l & 7.7 & 15 & 5.9 & 3 & 2.2 & 9.8 & 1.4 & 45 \\
\hline Rnps1 & 4.7 & 13.5 & 9 & 6.6 & 3 & 2.7 & 4.5 & 44 \\
\hline Phactr4 & 7.1 & 2 & 4.7 & 5.1 & 5.7 & 2.8 & 15.8 & 43.2 \\
\hline Ralgps1 & 3.8 & 14.6 & 4.8 & 10.1 & 5.2 & 2.6 & 0.6 & 41.7 \\
\hline Nebl & 11.8 & 1.7 & 7.7 & 3.5 & 0.5 & 0.1 & 15.2 & 40.5 \\
\hline Phc3 & 10.5 & 5.9 & 8.4 & 7.4 & 1.9 & 1.7 & 3.5 & 39.3 \\
\hline Mpv17I & 2.9 & 9.9 & 6.1 & 8.6 & 6.7 & 0.7 & 1.1 & 36 \\
\hline Med12l & 14.9 & 3.6 & 5.8 & 2.4 & 0.3 & 4.3 & 0.1 & 31.4 \\
\hline Cast & 0.5 & 0.8 & 0.5 & 0.1 & 0.1 & 0.7 & 27.2 & 29.9 \\
\hline Tead1 & 14.6 & 8.4 & 2 & 0.5 & 0.1 & 0.1 & 3.7 & 29.4 \\
\hline Rbm34 & 3.8 & 5.5 & 2.9 & 3.7 & 1.5 & 1.3 & 1.9 & 20.6 \\
\hline Vwa5b2 & 0.1 & 10.1 & 2 & 1 & 2.6 & 0.7 & 0.1 & 16.6 \\
\hline Cacna1e & 0.3 & 5.6 & 6 & 1.1 & 0.1 & 0.1 & 2.9 & 16.1 \\
\hline Col20a1 & 0.7 & 0.6 & 3 & 9.7 & 1.6 & 0.2 & 0.2 & 16 \\
\hline Detd & 4.5 & 1.7 & 4.7 & 1.5 & 0.2 & 0.5 & 1.7 & 14.8 \\
\hline Hs6st2 & 0.3 & 7 & 4.8 & 0.8 & 0.1 & 0.1 & 0.2 & 13.3 \\
\hline Morn1 & 1.3 & 1.4 & 0.7 & 1.3 & 1.5 & 0.4 & 0.9 & 7.5 \\
\hline Klhl29 & 0.1 & 3.8 & 0.9 & 0.5 & 0.3 & 0.2 & 0.1 & 5.9 \\
\hline Ubxn11 & 1.3 & 1.5 & 0.4 & 0.8 & 0.7 & 0.6 & 0.2 & 5.5 \\
\hline Zfp11 & 1.9 & 0.5 & 1 & 0.7 & 0.3 & 0.1 & 0.3 & 4.8 \\
\hline Afmid & 0.9 & 0.9 & 0.5 & 0.4 & 0.2 & 1.4 & 0.4 & 4.7 \\
\hline
\end{tabular}

Figure 4. 40 DAS genes (Figure 3), annotated according to Brain Cells Atlas (Zhang et al., 2014), elucidating glial-specific genes. Blue color of gene name (first column) corresponds to microglia; green-endothelial cell; orange-astrocyte. Double colors signify both cells maintain expression of the corresponding gene. Uncolored gene names correspond to other (non-glial) cell types. "Od." abbreviation stands for "Oligodendrocyte". Genes sorted descendent for overall expression rate (last column). 


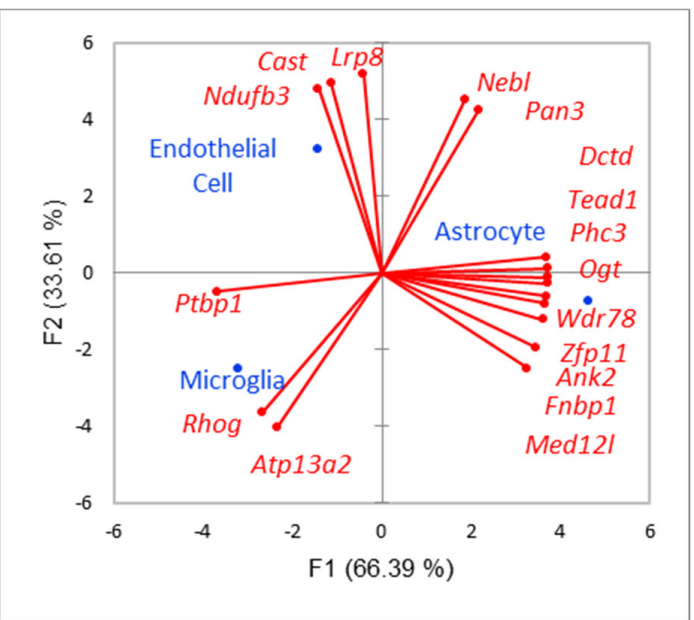

Figure 5. Selected 17 glia specific DAS genes.

\subsection{Microglial Genes}

We annotated Ptbp1, Rhog, Atp13a2, and Afmid according to Table 1.

\subsubsection{Rhog Gene}

Rhog (Ras homolog gene family, member g (rho g)) coordinates response to 'bacterial invasion of endothelial cells' as is underlined by GO enrichment term (Figure 6).

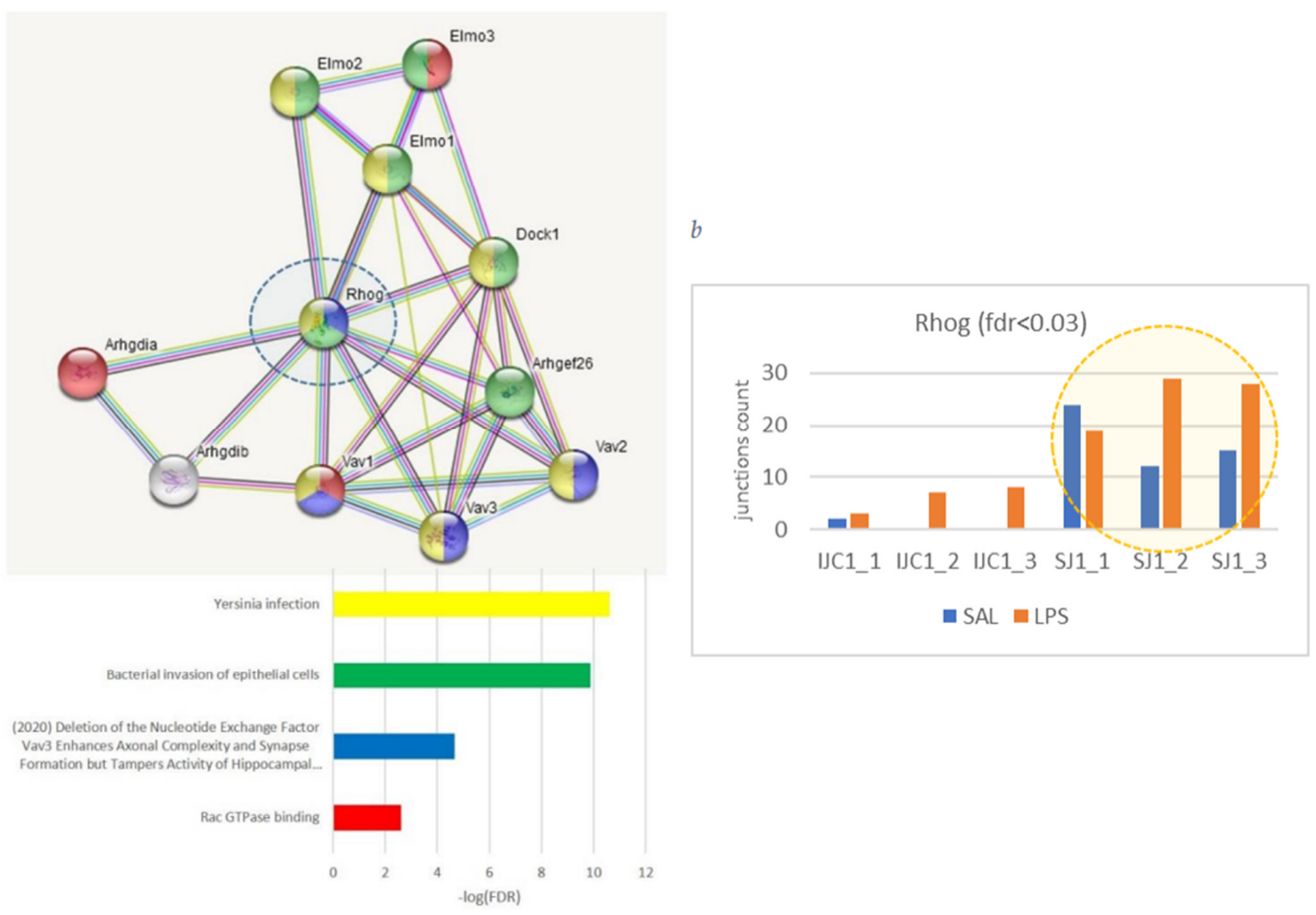

Figure 6. (a) Rhog genetic environment recovered by string-db.org facility. We observe it involvement in 'Bacterial invasion of epithelial cells' GO term, implying distinct LPS response. (b) Both Rhog 5' UTR isoforms elevated (DAS FDR < 0.03); denotation: IJC ('include junction counts'; Shen et al. 2014) stands for read counts overlapping exon inclusion instances for three replicas per group (ijc1, ijc2, ijc3); SJC ("skipped junction counts") stands for read counts overlapping exon skipping junctions for each of three species per group. 
We observed Rhog DAS ES event as 5' UTR exon 4 skipping (Figure 6; right panel) implying it expression elevation in LPS group (Figure 3). Additionally, we observed distinct elevated expression of long isoform in LPS compared to SAL group, along with higher expression of short one (Figure 6; right panel). Exon 4 skipping is manifested as a major isoform in both SAL/LPS groups (Figure 6).

Figure 7 demonstrates non-linear coordinated elevation/downturn of Atp13a2, Afmid isoforms expression (Figure 3).
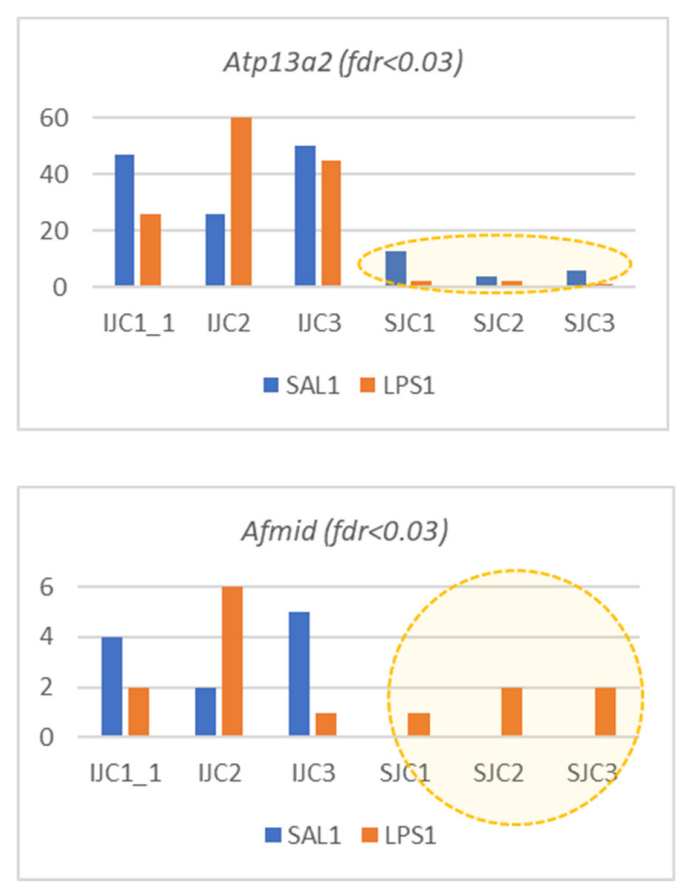

Figure 7. DAS ES events of microglial genes given Atp13a2 increased expression in LPS, while Afmid decreased its expression (Figure 3). Short isoform is noncoding in both cases.

\subsubsection{Ptbp1 Exon 8 Skipping Preference}

Strikingly, we also observed significant exon 8 skipping in neurospecific splicing factor, Ptbp1 (Figure 8), responsible for splicing alteration of several thousand genes [23]. Along with its expression elevation in LSP (Figure 9), its splicing skews towards to exon 8 skipping (Figure 5) implies putative immunomodulation in astrocytes/microglia/endothelial cells in LPS1 species, since Ptbp1 short isoform weakens its polypyrimidine tract binding [23]. As exon 8 skip in Ptbp1 alters target exon inclusion rate based on Ptbp1 binding preference [23], this way altering exons inclusion rate (both up and down), we may speculate it serves as a switch to turn on immune response by altering exon inclusion/skipping events ratio in specific genes.

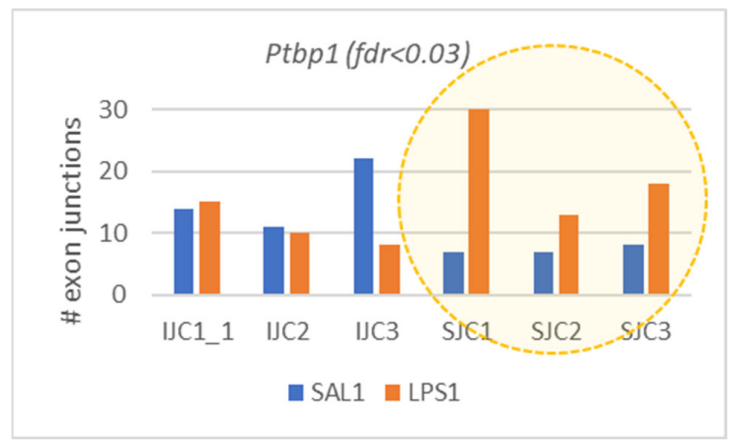

Figure 8. Alteration of major isoform from long to exon eight skipped one in LPS group, regarding to in-frame exon 8 (DAS FDR <0.03). 


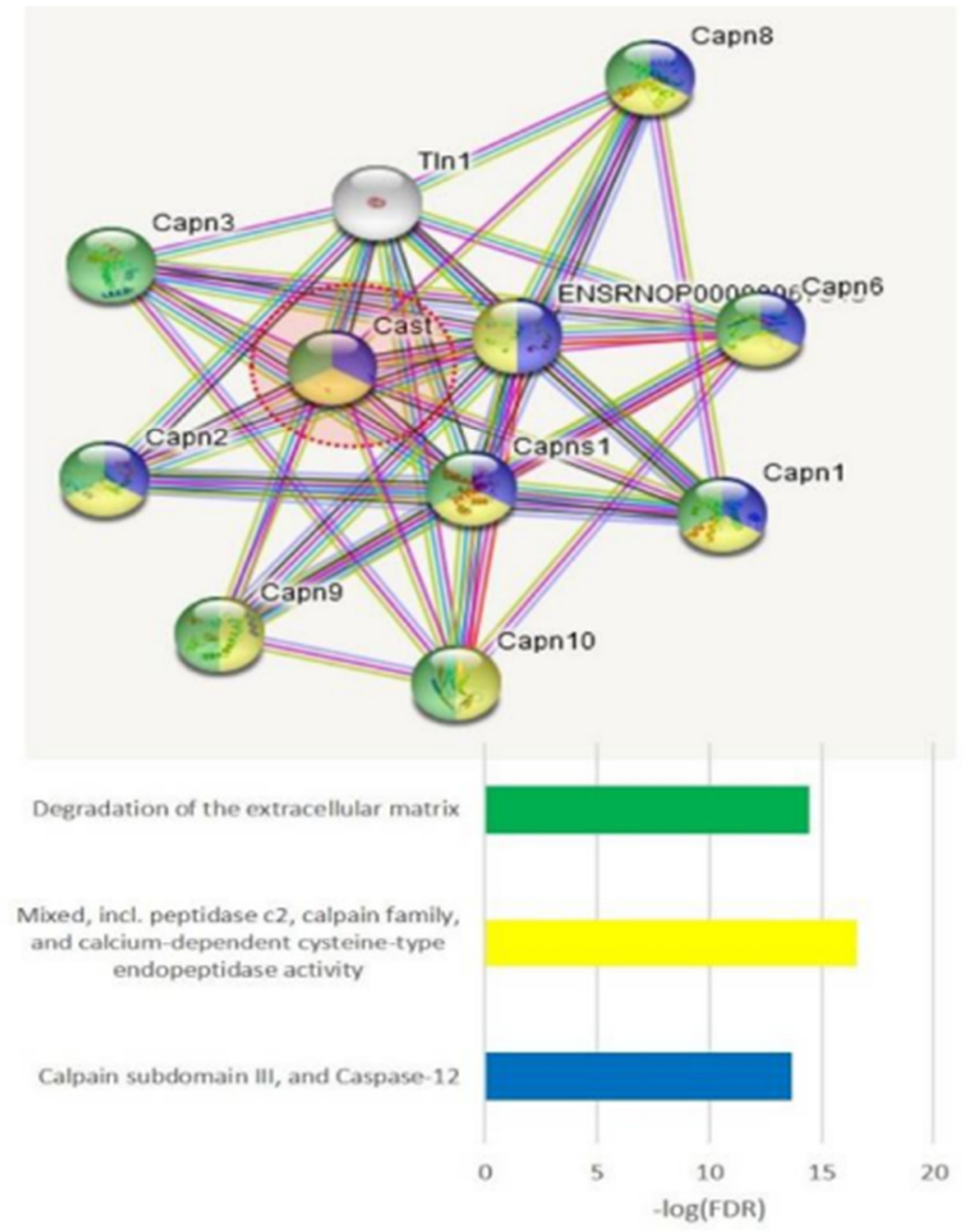

$b$

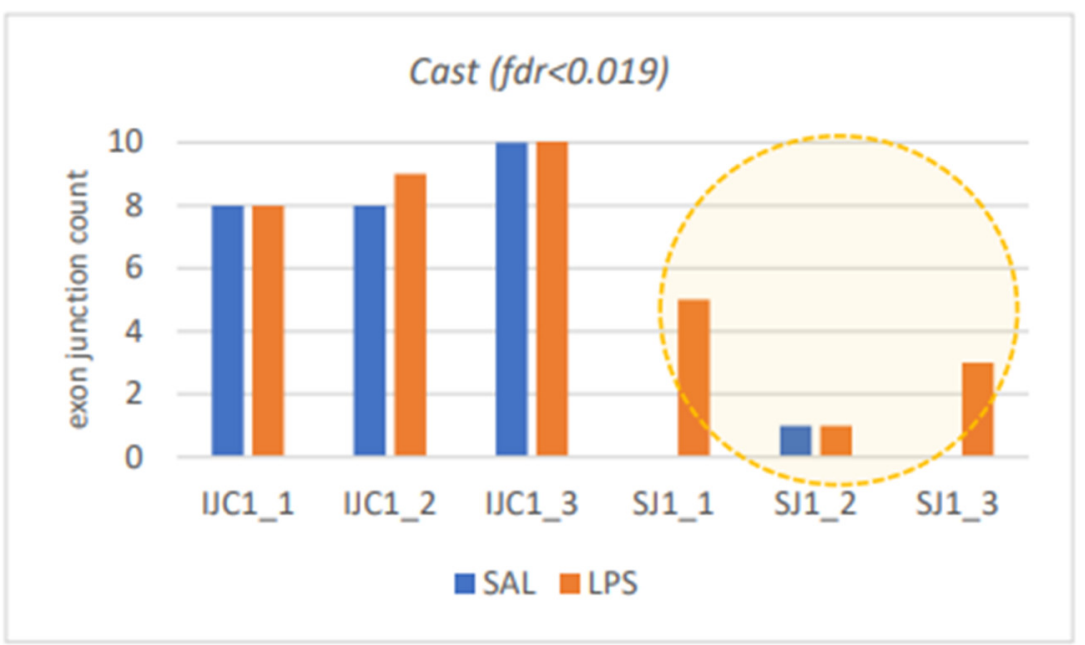

Figure 9. (a) Neighborhood environment of Cast and GO annotation. (b) Observed expression elevation of skipped isoform in LPS group. 
Based on annotation of 4 microglial cell specific genes we may state that DAS underscores specific major isoform enhancing. Three genes (Ptbp1, Atp13a2, and Rhog) have their expression rate increased along with altering isoforms ratio (Figure 3), while Afmid decreased its expression in LPS (Figure 3) featuring skipping of coding exon and thus being a subject to nonsense mediated decay (NMD) due to ORF disruption.

\subsection{Endothelial Specific DAS Genes}

Herein we annotate the endothelial cells specific DAS genes (Table 2): Cast, Phactr4, $\mathrm{Nebl}, \mathrm{Lrp} 8$, and Pkn2. Figures 9-13 underline the relevance of DAS genes to immune modulated features.

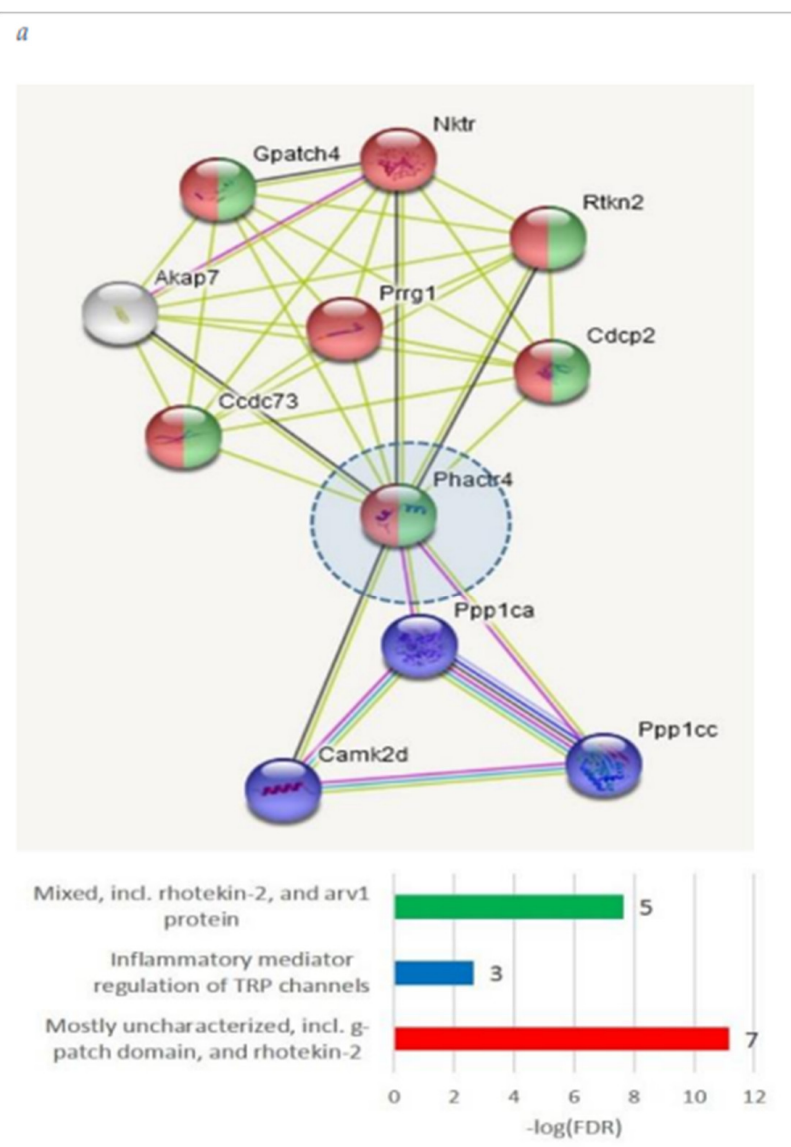

$b$

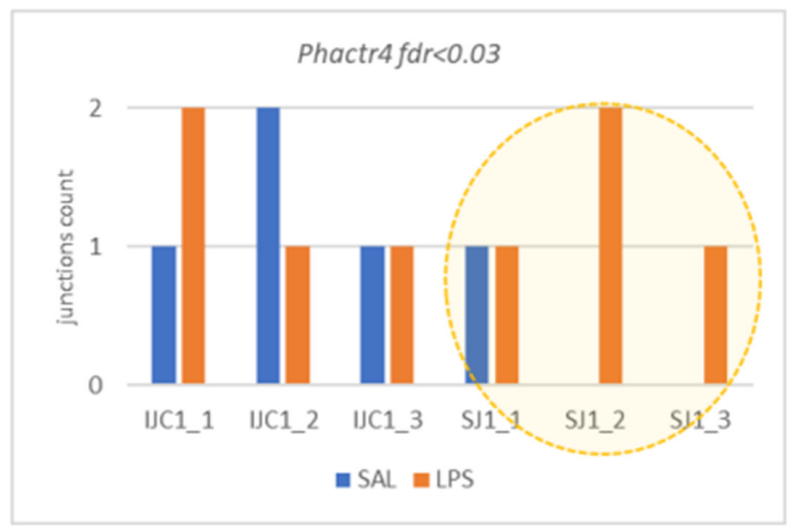

Figure 10. (a) String-db gene environment implies Phactr4 mediating the inflammatory regulation of TRP (transient response potential) channels; (b) Short isoform is distinctly elevated in LPS group (Figure 3). 
Table 2. Key SAL_LPS DEG networks inferred by GO annotation.

\begin{tabular}{cccc}
\hline GO_id & Definition & Obs/Exp & FDR \\
\hline GO: 0002366 & Immune system process & 25 of 945 & $9.70 \times 10^{-23}$ \\
GO: 0006955 & Immune response & 19 of 506 & $8.32 \times 10^{-19}$ \\
GO: 0007165 & Signal transduction & 25 of 2142 & $1.03 \times 10^{-14}$ \\
GO: 0071222 & Cellular response to lipopolysaccharide & 10 of 153 & $2.39 \times 10^{-11}$ \\
GO: 0001819 & Positive regulation of cytokine production & 10 of 239 & $1.18 \times 10^{-09}$ \\
GO: 0001817 & Regulation of cytokine production & 15 of 390 & $1.84 \times 10^{-08}$ \\
GO: 0032496 & Response to lipopolysaccharide & 14 of 306 & $1.26 \times 10^{-08}$ \\
\hline
\end{tabular}

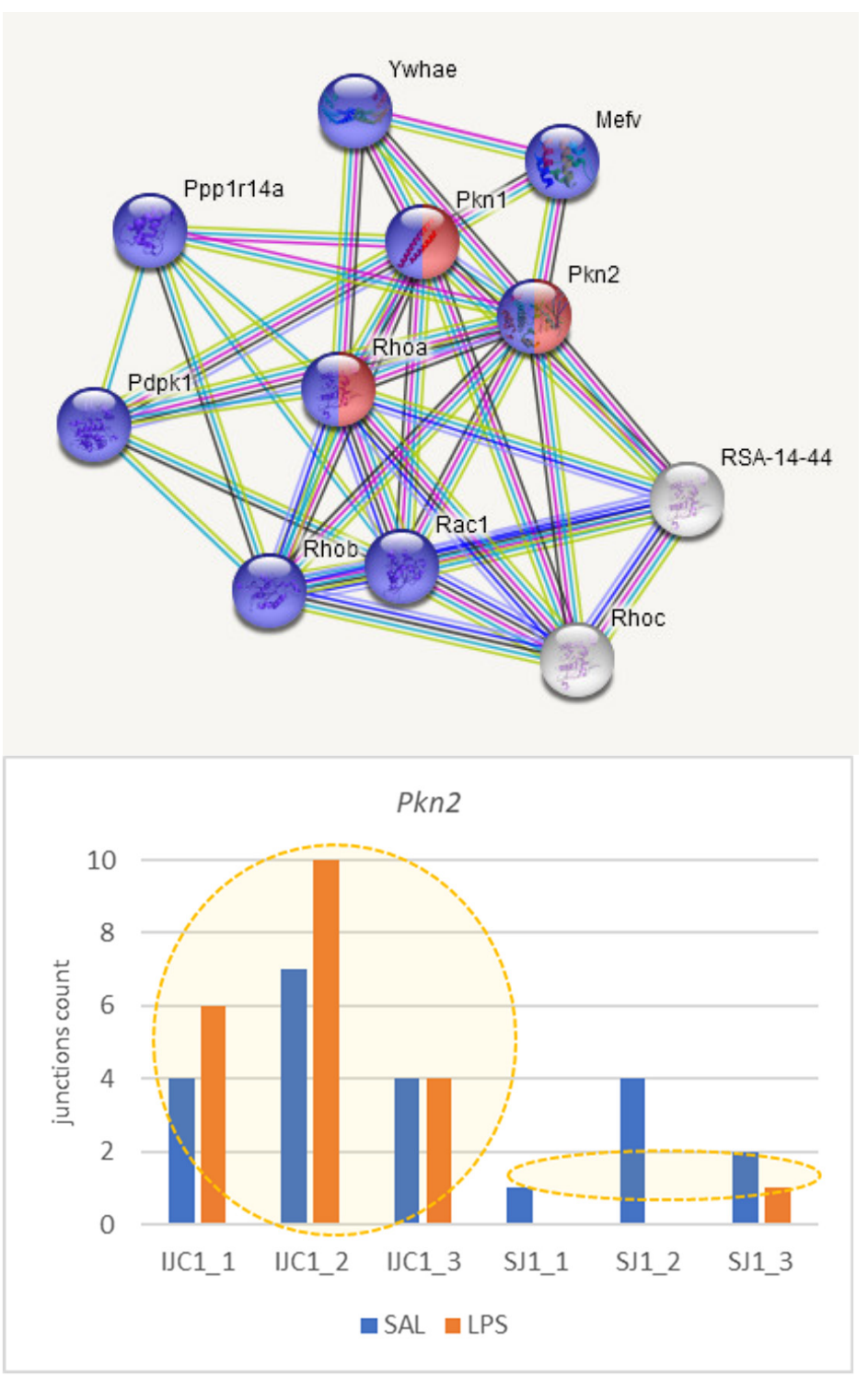

Figure 11. Pkn2 is involved in 'epithelial cells migration' cascade (three genes, red; GO:0010631; enrichment FDR < 0.0017) and is a part of 'Response to stimulus' network (nine genes, blue; GO:0050896; enrichment FDR < 0.0037). DAS FDR < 0.03, AS enhances inclusion of coding exon in LPS sample. 

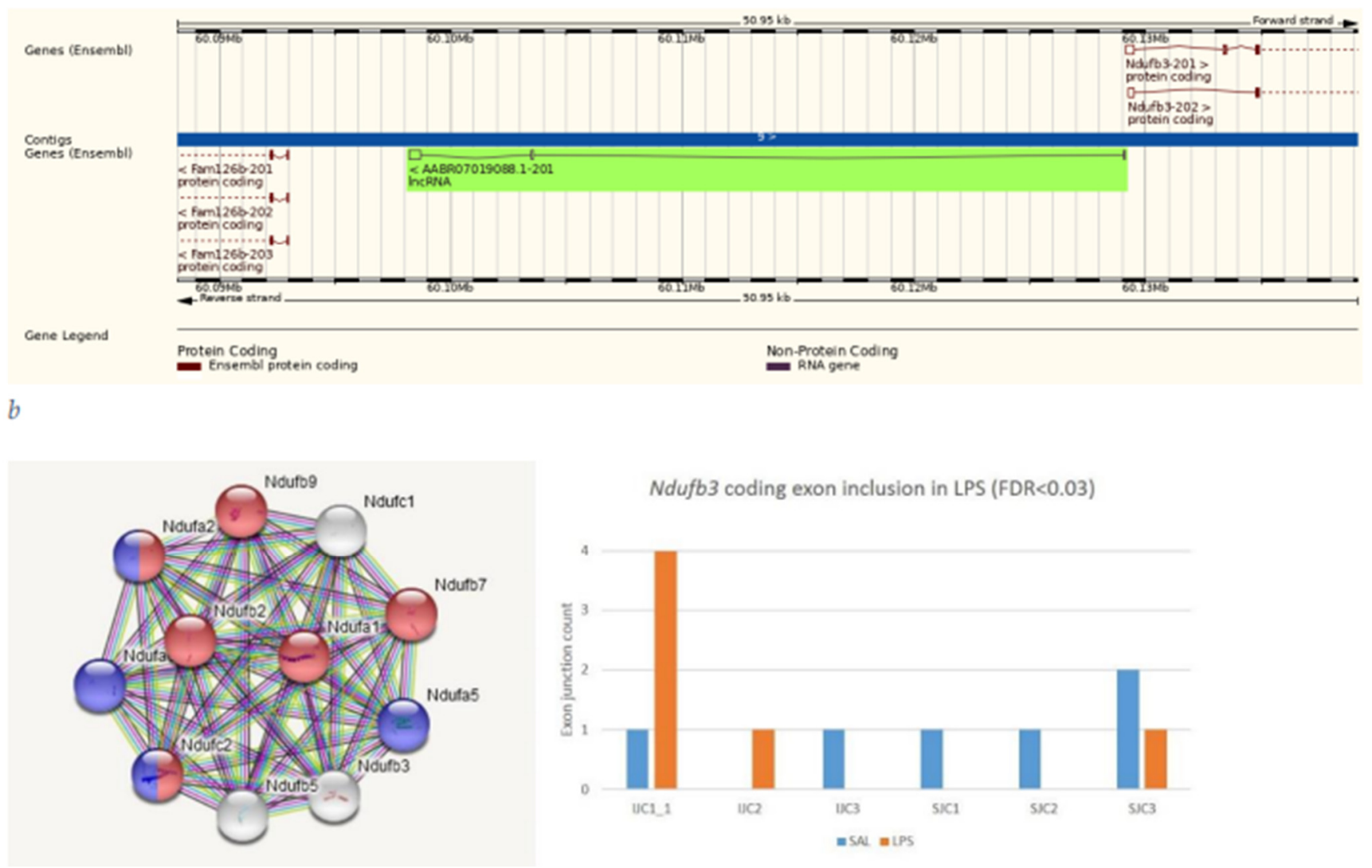

Figure 12. (a) Noncoding RNA $A A B R 07019088.1$ manifests exon 2 elevated insertion (DAS FDR < 0.04; Table S3) in Nduf3b antisense location along with Ndufb3 one in LPS group; (b) Oxidoreductase MWFE subunit mitochondrial related gene $N d u f b 3$ switched TSS by coding exon inclusion (see (a)) in LPS group enhancing functional expression. Color coding: Red: GO: CL:22493; description: ubiquinone, and NADH-ubiquinone oxidoreductase MWFE subunit: obs vs. exp: 6 of 8; FDR: $2.53 \times 10^{-12}$. Blue: GO: KW-0679, Respiratory chain; obs/exp: 4 of 40; FDR $<1.83 \times 10^{-6}$.

\subsubsection{Cast Gene}

Cast is calcium-dependent cysteine protease. Based on annotation (Figure 9), we may state that cast is involved in 'degradation of extracellular matrix' and may be implicated in immune response processes.

\subsubsection{Phactr4 Gene}

Phactr4 gene was shown to be involved in mediation of transient response potential regulation upon inflammatory response (Figure 10a) featuring short isoform expression elevation in LPS sample (Figures 3 and 10b).

\subsubsection{Pkn2 Gene}

$P k n 2$ (Serine/threonine-protein kinase N2) is involved in endothelial cell migration (GO:0010594), also featuring intracellular signal transduction in response to stress (Figure 11). DAS ES event in this gene features its activation by elevation major long isoform in LPS group leading to its enhanced expression (Figure 3). 

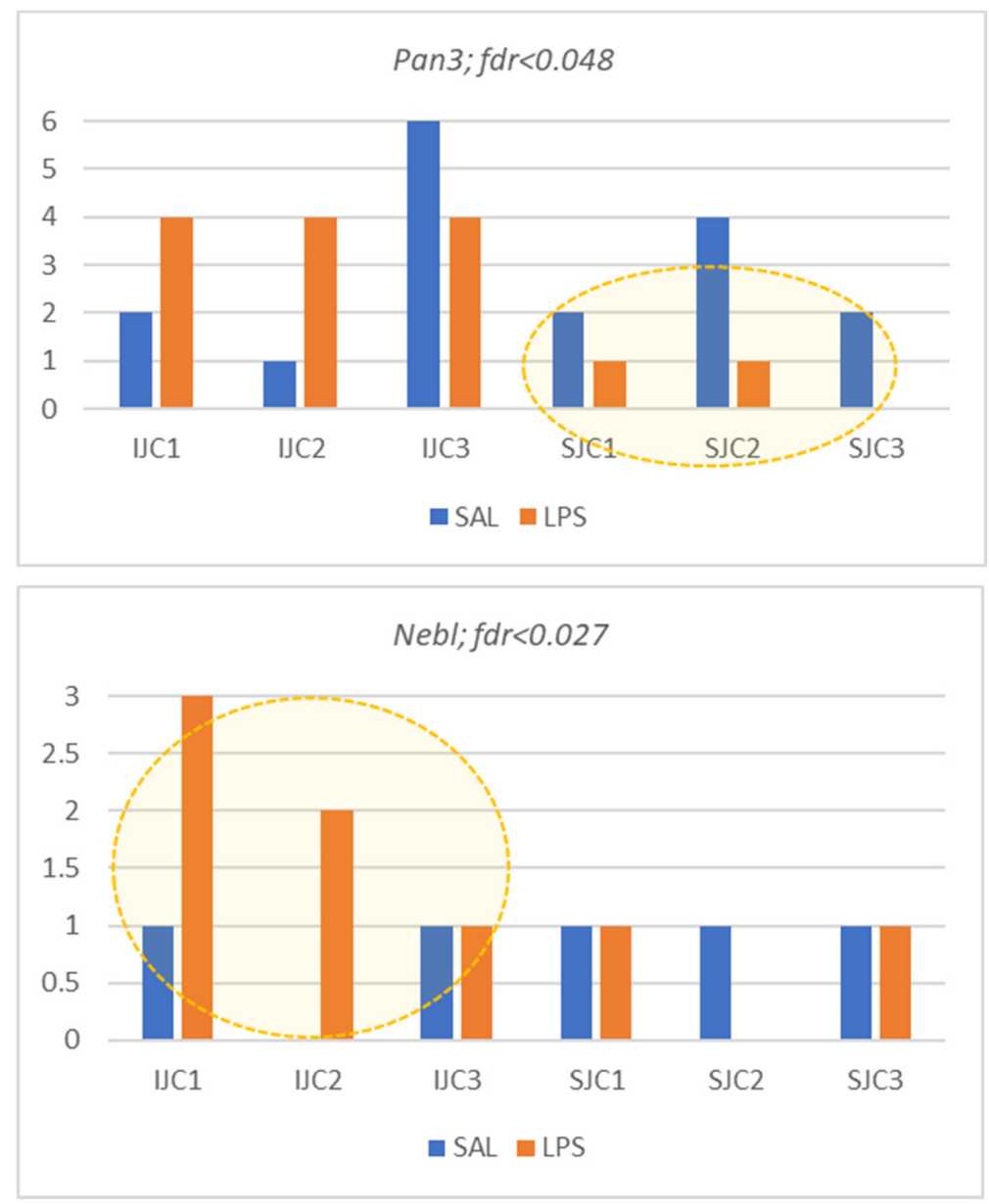

Figure 13. Both genes manifest elevated expression of long isoform in LPS (Figure 3) and attenuated expression of skipped (noncoding) ones.

\subsubsection{Ndufb3 vs. AABR07019088 Genes}

$\mathrm{Ndufb3}$ is a member of MWFE subunit of the mitochondrial NADH-ubiquinone oxidoreductase (complex I) is a small, essential membrane protein of 70 amino acids, which is made in the cytosol, imported into mitochondria, and assembled without further proteolytic processing. AABR07019088 is antisense RNA of Ndufb3 (Figure 12a). Both genes elevated their long isoforms expression in LPS group (Figures 3 and 12b), Ndufb3 isoform featuring alternative translation start site (TSS; Figure 12a).

\subsubsection{Pan3 and Nebl Genes}

Pan3 (Poly(A) Specific Ribonuclease Subunit) and Nebl (Nebulette; Actin-Binding Z-Disk Protein) are housekeeping genes. Long isoforms elevation (Figure 13) may witness enhanced metabolism in LPS group (Figure 3).

\subsection{Astrocyte Specific DAS Genes}

Astrocyte specific DAS genes: Ank2, Med12l, Tead1, Milt10, Fnbp1, Zfp1, Wrd78, Eogt, $D c t d, P h c 3$, and Mok (Table 2) manifest the largest group, with two genes (Ank2, Eogt) maintaining the highest DAS significance.

We observed the two most significant DAS ES events (Table S3, Supplementary Materials) in Ank2 (DAS FDR < 0.006) and Eogt (DAS FDR <0.00014) genes altering its major isoforms upon LPS exposure (Figure 14). Both these genes also express in neurons (Figure 4). Notably, while Ank2 increased its expression in LPS, Eogt was attenuated (Figure 3), due to $5^{\prime}$ UTR related exon skipping implying glycosylation process downturn. 

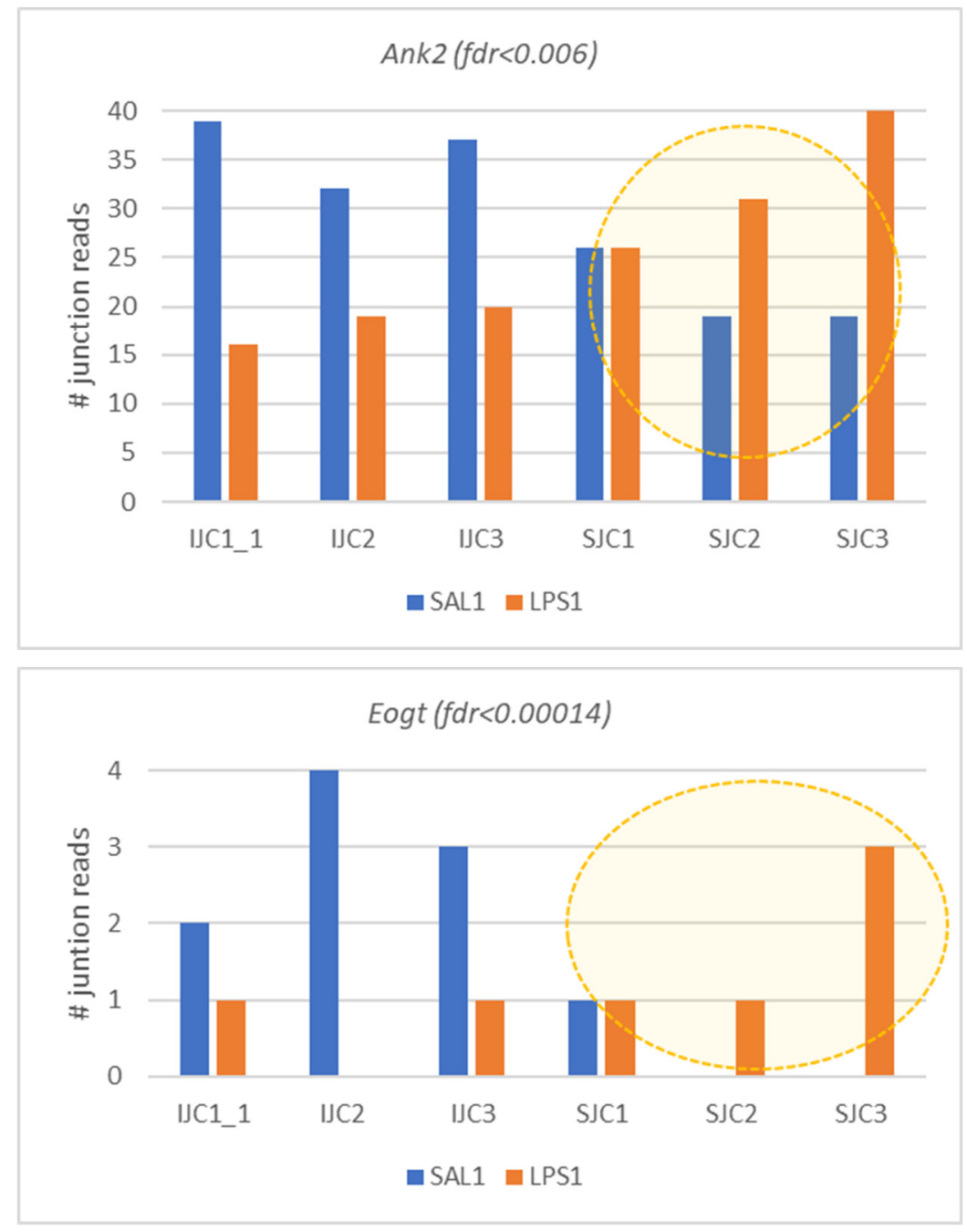

Figure 14. Switching major isoforms in two highly significant DAS genes. Ankyrine 2 (Ank2) gene increases short isoform expression upon LPS exposure, while Eogt Glycosyltransferase gene expressed, mostly in astrocytes, is switched off by DAS altering $5^{\prime} \mathrm{UTR}$ region.

The rest of DAS genes referring mainly to transcription process and apparently featuring metabolism intensity increase in LPS (Figure 3), all manifesting long isoforms in LPS group, are presented in Figure 15.

\section{Fnbp1 Gene}

Fnbp1 is Formin binding protein 1. It is required to coordinate membrane tubulation with reorganization of the actin cytoskeleton during the late stage of clathrin-mediated endocytosis. (DAS FDR < 0.027). We observed its major isoform elevation, accompanied with attenuation of alternative skipped isoform (Figure 16).

\subsection{Splicing Factors (SF) in Brain}

We assessed 15 key SFs expression in the brain using Barres Lab atlas for mature brain cell specific expression [22]. As can be seen from Figure 17, the majority of brainspecific SFs are neuron specific ones, as was reported earlier, in a range of publications starting from 2005 [24,25]. Still, there is a range of SFs, in particular, Ptbp1, expressing preferentially in microglia and endothelial cells (Figure 13). As we observed, Ptbp1 exon 8 skipping as highly specific for LPS exposed species (Figure 4), we speculated it might affect immunomodulation in response to LPS. 

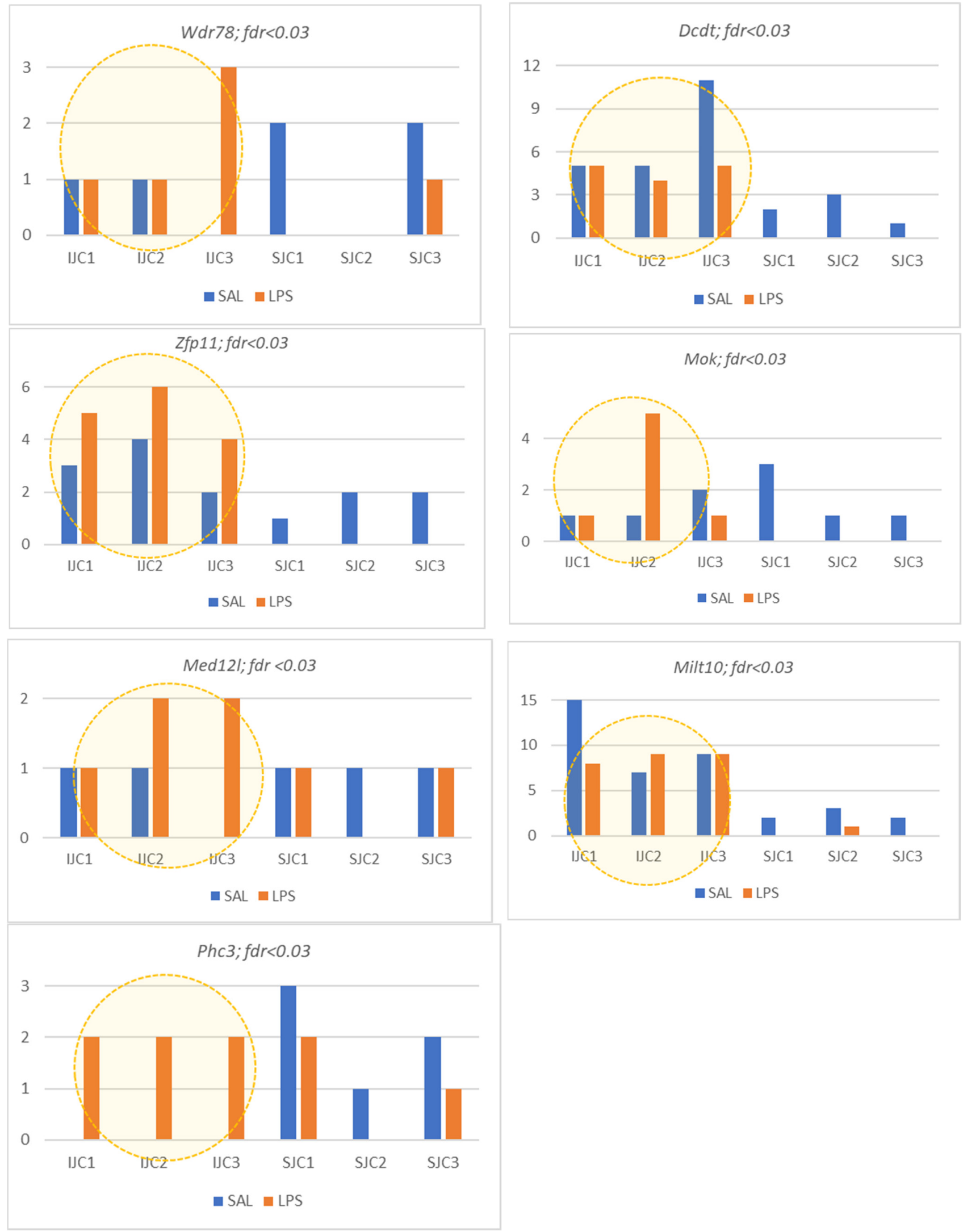

Figure 15. Panel of seven astrocyte specific low expressed DAS ES events. (Wdr78, Dynein Axonemal Intermediate Chain 4; Dctd, Deoxycytidylate Deaminase; Zfp11, transcription factor; Mok, MOK protein kinase; Med12L, Mediator Complex Subunit 12L; Mllt10, Histone Lysine Methyltransferase DOT1L Cofactor; Phc3, Polyhomeotic Homolog 3). 


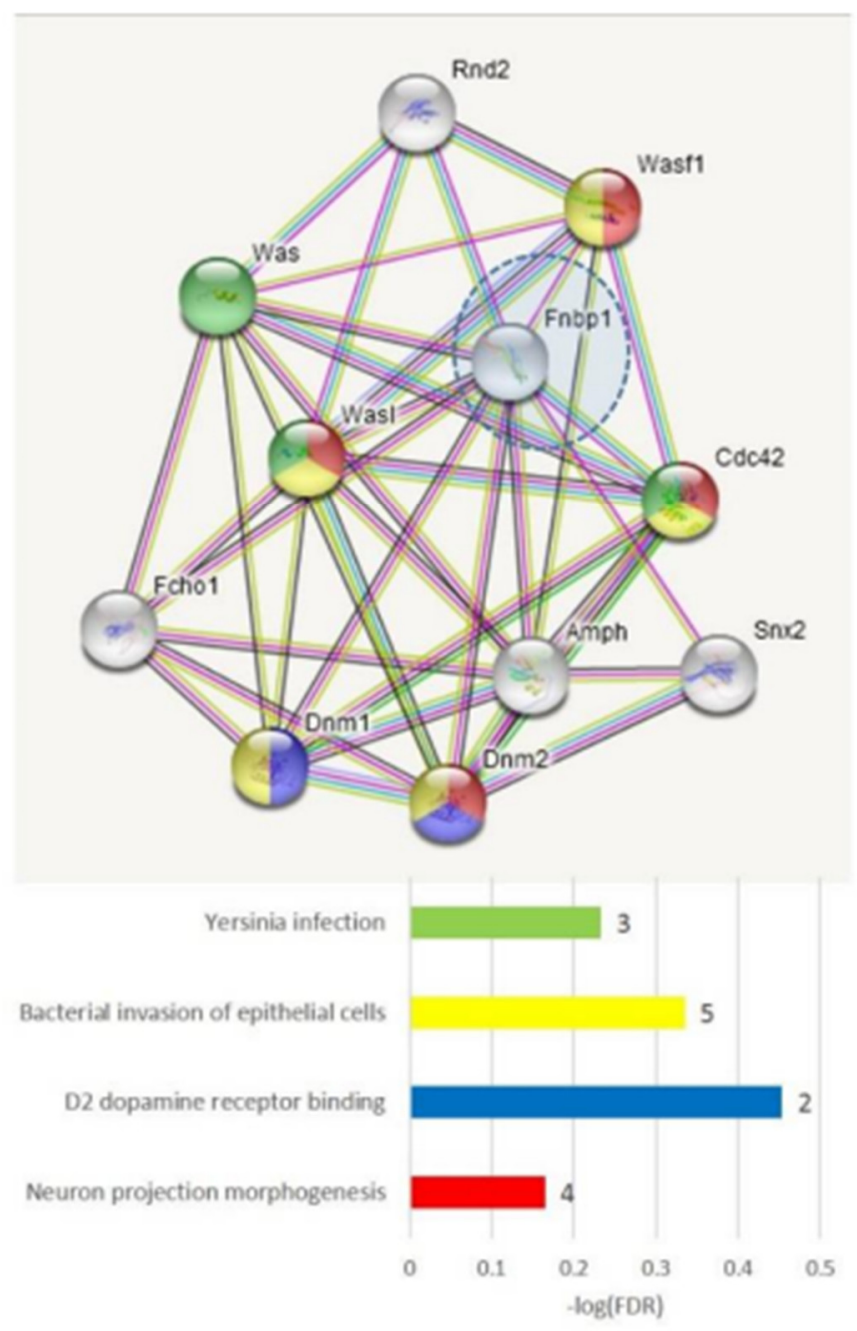

b

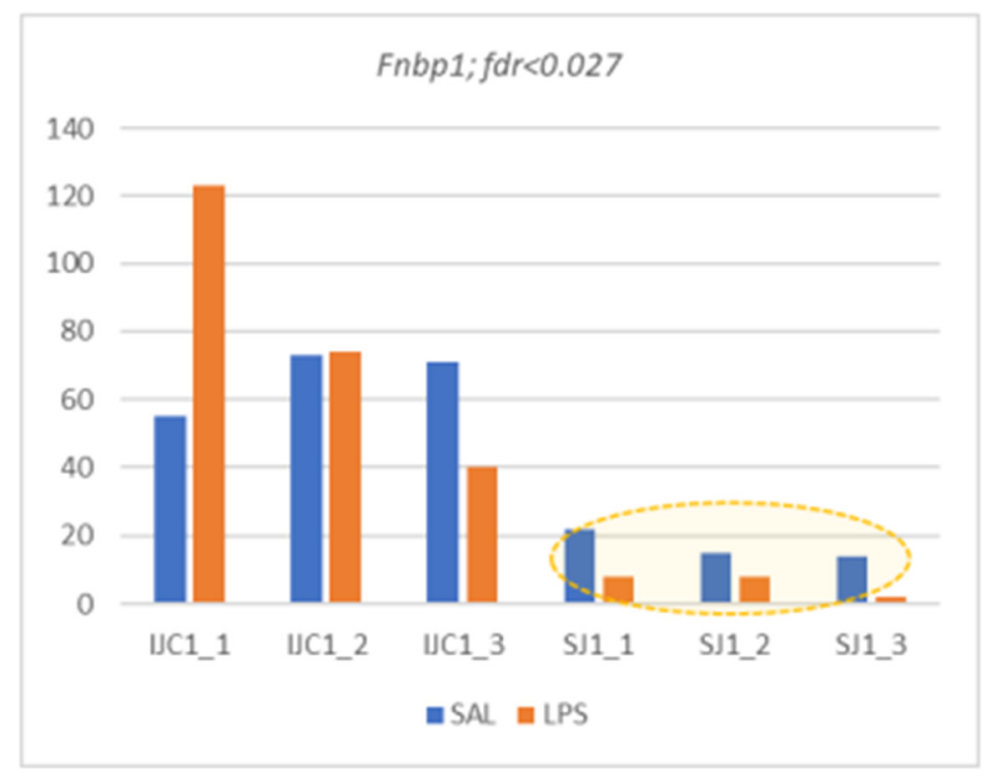

Figure 16. (a) Fnbp1 mediates 'Bacterial invasion of epithelial cells' response as well via specific networks. (b) We see attenuation of short isoform in LPS group (DAS FDR <0.027). 


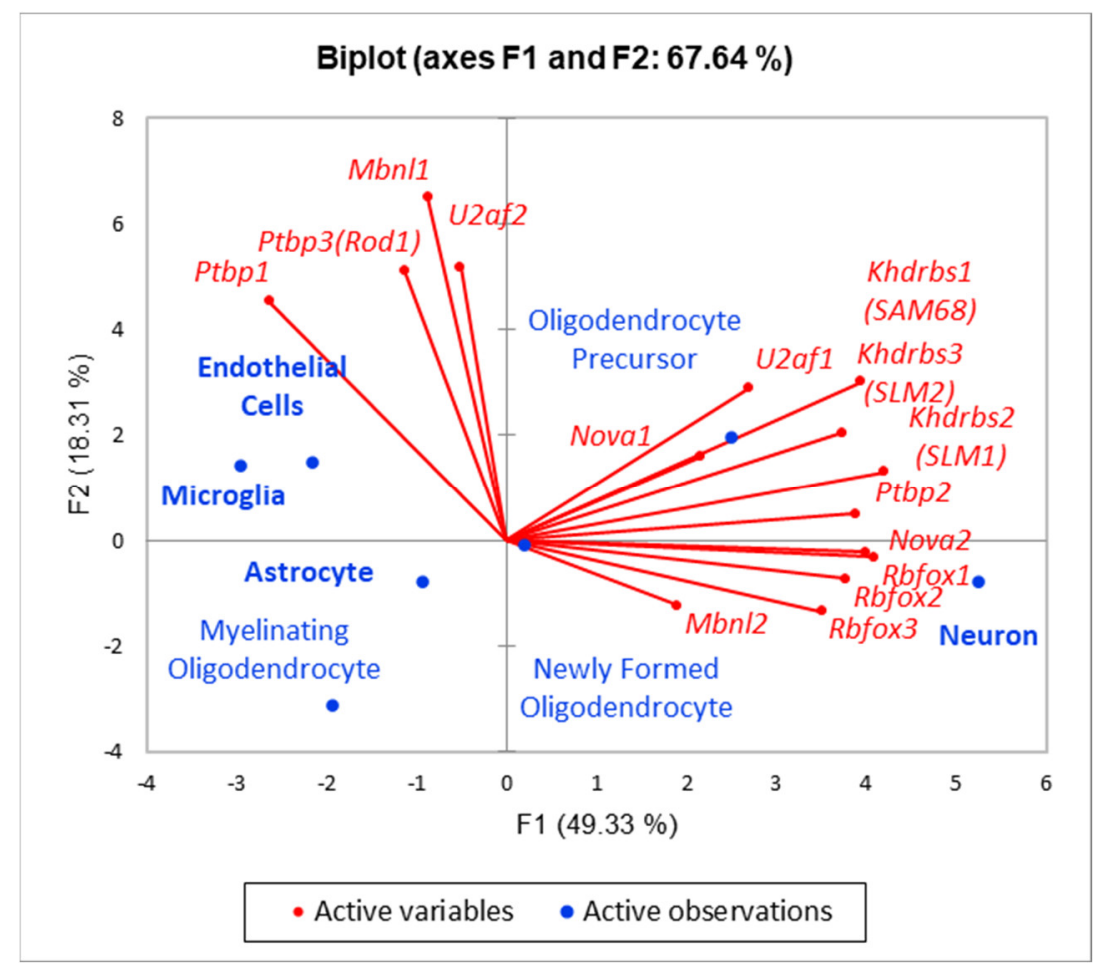

Figure 17. SFs expression preference in seven brain cell types [22]. Besides mostly neuron specific SFs (Nova, Ptbp2, Rbfox, SLM1, and 2) we may see four SFs preferential ones in glial cells (Ptbp1, Rod1, Mbnl1, and U2af2).

From Figure 18 we may state that glia specific SFs (Figure 18a) are elevated in LPS sample (Figure 18b), implying its involvement in LPS response.
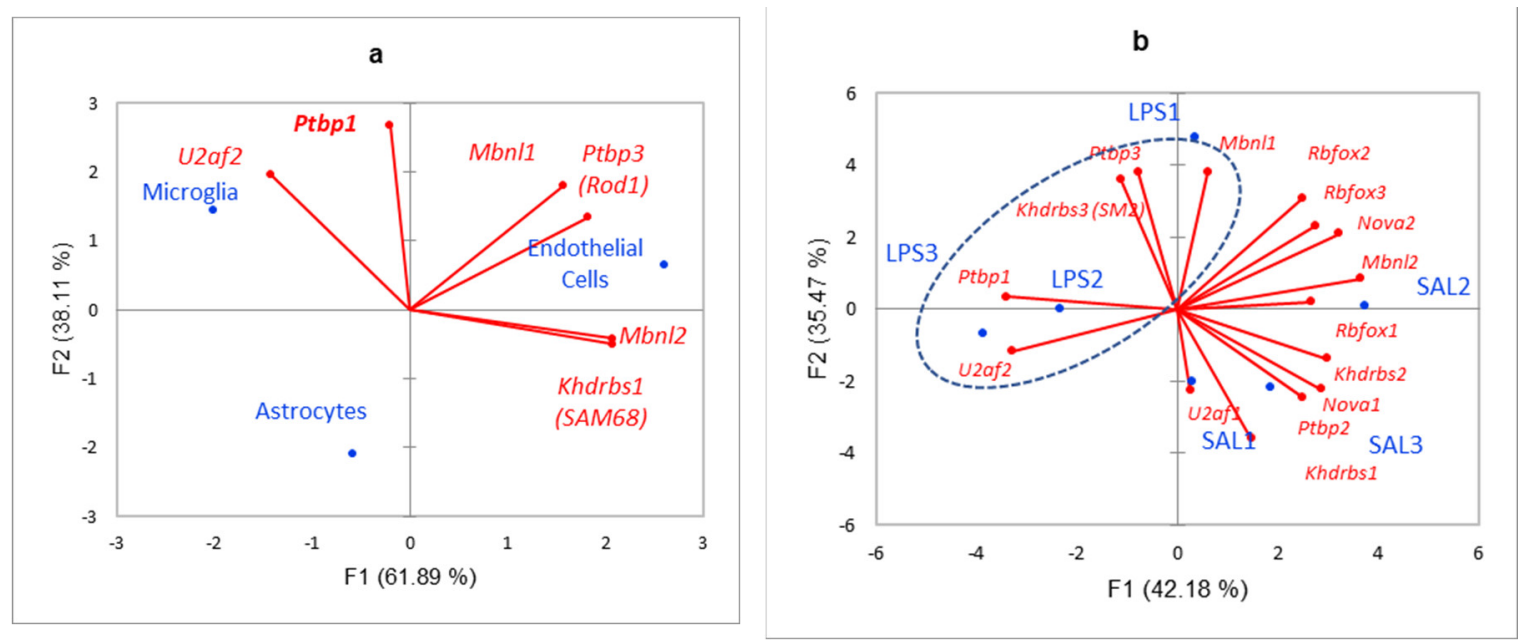

Figure 18. (a) PCA plot elaborating on glia specific SFs [22] underlined in Figure 17 across three glial cell types point to microglial and endothelial cells SFs activity, while astrocytes essentially lack SFs considered; (b) PCA plot of SFs expression profiling across 6 species underlining expression elevation of glial SFs specifically in LPS1 (encircled by blue oval).

\section{Discussion}

We conducted RNA-seq analysis of rat hippocampal tissues upon LPS exposure compared to saline administered ones. Analysis revealed 609 genes with significant difference in expression rate at $q$-value $<0.05$, which were further filtered with $\log 2$ fold $>2$ criterion, due to surgical invasion procedure in both SAL/LPS groups and consequent background 
inflammation in both groups (Table S1, Supplementary Materials). That left 93 DEGs that were subject to gene ontology annotation, yielding $284 \mathrm{GO}$ biological process terms. Using GOMCL toolkit [21], we elucidated single DEGs cluster based on ontology and consequent non-redundant 36 DEGs, encompassing all GO terms present in initial sample.

We present the key relevant events in Table 2, while full GO annotation can be found in Supplementary Table S2, Supplementary Materials. Based on Table 3 we may state we witness quite clear LPS effect within our sample compared to SAL control group. The 3 major clusters of coordinated genes.

Table 3. Minimal (a) and maximal (b) expression rate (TPM) of Ptbp1 major isoform (ES9) in 10 tissues (source: GTEX v.7). Bracketed is top/second isoforms ratio. Median Ptbp1 expression value across 53 tissues is 29.5 TPM.

\begin{tabular}{|c|c|c|c|c|}
\hline $\begin{array}{c}\text { (a) } \\
\text { Pancreas }\end{array}$ & Liver & Whole Blood & $\begin{array}{l}\text { Heart-Left } \\
\text { Ventricle }\end{array}$ & $\begin{array}{c}\text { Brain-Frontal } \\
\text { Cortex (BA9) }\end{array}$ \\
\hline $\begin{array}{c}9.4(1.01) \\
\text { (b) }\end{array}$ & $8.42(1.7)$ & $7.39(3.1)$ & $6.28(1.6)$ & $6.72(4.5)$ \\
\hline $\begin{array}{c}\text { Adipose- } \\
\text { Subcutaneous }\end{array}$ & Lung & $\begin{array}{c}\text { Cells- } \\
\text { Transformed } \\
\text { Fibroblasts }\end{array}$ & $\begin{array}{l}\text { Cells-EBV- } \\
\text { Transformed } \\
\text { Lymphocytes }\end{array}$ & $\begin{array}{l}\text { Cervix- } \\
\text { Ectocervix }\end{array}$ \\
\hline $82.4(3.1)$ & $85.5(2.6)$ & $80.5(2.4)$ & $101.4(4.5)$ & $57.3(1.9)$ \\
\hline
\end{tabular}

Based on DEG analysis, (Figures 1 and 2) we state that LPS response is quite robust (612 DEGs overall) and single directed (majority of DEGs are elevated in LPS group; Figure 2). The 36 core DEGs co-varied clusters are depicted in Figure 2. We should stress that the majority of DEGs are highly expressed glial genes. The cell specific analysis reveals the majority of DEGs are microglial specific ones following endothelial and astrocyte cells (Figure 2e,f).

Given AS in brain primarily refers to neurons primarily expanding their synaptic plasticity [26] and has been widely addressed therein; we decided on assessing AS-mediated LPS response specifically in glial cells for gaining the insight on it. Analysis of alternative splicing reveals a group of 50 structural and signaling genes being differentially alternatively spliced (see Table S3, Supplementary Materials). No particular abundance in GO categories were inferred for the DAS gene set. Still, while expanding the gene neighborhood of DAS genes there were relevant DAS genes networks affecting rho-gtpase activity, platelet-related genes (F8, wVag1; [27]) and a range of others (Figures 6-16).

With that, we should outline the basic challenges of splicing analysis we have observed. Due to relatively low read coverage in our study (about $30 \mathrm{mln}$ reads per transcriptome), we might miss some DAS genes, along with maintaining DAS low confidence (sensitivity) in a range of cases. Recently reported tandem splice sites (TASS) persistence reported in RNA-seq data [28] were also observed in our data quite abundantly (about 1.15 thousand, across 10,000 ES events total average per sample) given only $20 \%$ proved being nonspurious/sensible splice sites [28], also distorted the overall picture. We applied the filter of minimal exon junction counts to be more than five, totaling both groups, compared.

Additionally, based on our preliminary assessments with protein database, at least half of the ES events maintain open reading frame (ORF) disruption and invoking nonsense mediated decay (NMD), thus being purely the means of prompt regulation of homeostatic balance of particular genes expression [29].

Another fundamental problem is that, in contrast to expression temporal course, the splicing dynamics manifests a much more velocity, implying there are large pools of unspliced transcripts in the pool [30] waiting for the splicing 'decision'. Splicing alteration may be performed in a matter of milliseconds and be restored back immediately, especially for many auto-/cross-regulated splicing factors and chromatin rearrangement machinery by utilizing NMD routine while altering (downing) gene expression would take relatively longer time. 
Functional interpreting ES events is also a keystone. We approached this subject at least by validating coding/non-coding transcript variants resulted in the course of ES events. We revealed that only 8 DAS genes (Ptbp1, Fnbp1, Med12l, MegF11, Cast, Phc3, Cep295, and Ank2) maintain both coding isoforms, 19 DAS genes maintain long coding isoform only, no genes with skipped isoform coding only, and 23 remaining DAS genes maintain ES events in $3^{\prime} / 5^{\prime}$ UTRs. Elaborating on coding ES renders protein interaction analysis and, while not being already annotated experimentally, is hard to exemplify. Still, new means of protein isoforms annotation are approaching with an advent of AlfaFold resource [31].

Ptbp1 is one of the key neurogenesis factors, also used in reprogramming neural cells [8,32-34] We elucidated it is primarily expresses in glial cells (Figure 4), while highly repressed in neurons. Intriguingly, recent study demonstrated morphing astrocytes into dopaminergic neurons when depressing Ptbp1 [34]. They did it with virus vectors while natural antagonist of Ptbp1 in embryogenesis is mir-124 [8]. With that, the highest expression of Ptbp1 is observed in microglia and endothelial cells.

As we found essential splicing factor Ptbp1 being significant DAS gene (FDR < 0.03) in our set, we decided exploring brain-specific splicing factors genes group, since these affect splicing the most and, while being altered and impact several thousand of relevant exons reported elsewhere $[5,8,23]$. Upon the analysis, we report glial SFs manifest augmented expression in LPS group (Figure 13) emphasizing their relevance.

The mammalian specific Ptbp1 exon 8 (9 in human) skipping (27aa) manifests one of current enigmas not fully apprehended. It reported to be involved in embryonic stage tuneup after NSC -> NPC stage splicing tune up [23]. It is reported therein "exon 9 possesses splicing regulatory activity that is partially separable from the repressive activity conferred by RRM2 and that skipping of exon 9 reduces the negative and positive regulatory activities of PTBP1 without substantially affecting RNA binding activity", implying significant effect of the ES9 event.

Notably, according to GTEX v.7 data (gtexportal.org), Ptbp1 ES9 short isoform (ENST00000349038; NM_031991) is a major one with average expression rate of 31 transcripts per million (TMP) across 53 tissues, while exon 9-in most abundantly expressed variant (ENST00000350092; 5' UTR truncated isoform) manifests 16 TPM average, with other 16 ones manifesting 3.5 TPM and less, still comprising overall around $40 \%$ of Ptbp1 total expression rate per tissue. There is only one coding ES9 isoform in human Ptbp1 isoforms spectra, except for extremely rare transcript ENST00000627714.2, which simultaneously lacks all RNA recognition motifs (RRMs) coding exons and not detected in GTEX data. Two top expressed Ptbp1 isoforms co-vary with Pearson $r=0.81\left(\mathrm{df}=52 ; p<1 \times 10^{-7}\right)$.

Based on analysis of Ptbp1 expression organism wide with GTEX human resource, we elucidated that it's a redundantly expressed gene observed in virtually all cells except for mature neurons, and immune competent organs including heart, whole blood, pancreas, liver (Table 3a). Oppositely, cancer-related tissues/cells manifest high Ptbp1 expression rates, as reported in studies [11,35]. Ptbp1 deletion enhances MHC II expression in dendritic cells [13], therefore attenuating its binding affinity by ES8 may do the work. Based on glial cells analysis, we may state that Ptbp1 is significantly expressed in epithelial cells, as well (Figure 4).

Given Ptbp1 is primarily a glia specific gene among differentiated brain cells, we found its overall expression elevated specifically in LPS administered group, though not significantly (Figure 3. $p$-value $<0.33$ ), at the same time featuring its major isoform, significantly altering toward ES8 one (Figure 8), implying some stress response activity. Expanded Ptbp1 isoforms spectra (17 entries) allows for proposing Ptbp 1 isoform expression ratios, which may impact the immunogenic potential of glial cells, judging by the dynamics it manifests upon LPS response in the hippocampus brain region. 


\section{Conclusions}

While many of DAS events relate to the modulation of expression rates (increasing/decreasing), we may underline the enhanced accuracy of splicing routine in the course of gene expression elevation (often non-significant one), since short isoform (usually noncoding one) preference often manifest attenuation in a vast range of cases. We report that, while DAS genes and DEGs do not overlap, as a rule (e.g., [36]), it was observed that NMD employed coding gene expression abrogation correlates with its expression rate downturn, implying certain feedback routines at the transcription layer [37,38]. To this end, we observe certain glial DAS genes change its expression rate in nonrandom manner (Figure 3).

While we cannot immediately, functionally interpret the AS ES events observed, besides overall expression rate alteration, we correspond that there are plenty of statistically significant AS ES alterations in glial genes, in particular. Underlining their abundance, further research may shed light on the functional meaning of DAS ES events, besides NMD-related ones. We stress the DAS of Ptbp1 as one of the potent events possibly leading to massive alteration of splicing landscape upon LPS administration.

Supplementary Materials: The following supporting information can be downloaded at: https: / / www.mdpi.com/article/10.3390/biom12020277/s1, Table S1: List of Differentially Expressed Genes in SAL vs. LPS groups, Table S2: GO Functional annotation, Table S3: 49 Differentially alternatively spliced (DAS) genes as reported by rMATS software http:/ / rnaseq-mats.sourceforge. net/download.html, accessed on 3 January 2022, [20], Table S4: Annotation of 49 DAS genes for number of alternative isoforms in NCBI vs authors' RNA-Seq isoforms number.

Author Contributions: Conceptualization: G.T.S. and N.N.D.; methodology: G.T.S. and V.N.B.; formal analysis: G.T.S. and V.N.B.; investigation: D.A.L., E.V.S. and N.N.D.; data curation: G.T.S. and V.N.B.; writing—original draft preparation: G.T.S. and V.N.B.; Writing—review and editing: G.T.S. and V.N.B.; project administration: G.T.S.; funding acquisition: G.T.S. All authors have read and agreed to the published version of the manuscript.

Funding: This study was supported by grant from the Russian Science Foundation (N 20-64-47013) and the state budget project (0259-2021-0015: care of animals).

Institutional Review Board Statement: The study was conducted according to the guidelines of the Declaration of Helsinki and approved by the ethic committee of the Institute of Cytology and Genetics in accordance with the guidelines of the Ministry of Public Health of Russia (supplement to order N 267 of 19 June 2003).

Informed Consent Statement: Informed consent was obtained from all subjects involved in the study.

Data Availability Statement: Data supporting reported results can be found in European Nucleotide Archive (ENA) with id: PRJEB50635.

Acknowledgments: The authors are grateful to MDPI editorial and production team for a big help with manuscript editing.

Conflicts of Interest: The authors declare no conflict of interest. The funders had no role in the design of the study; in the collection, analyses.

\section{References}

1. Radenovic, L.; Nenadic, M.; Ułamek-Kozioł, M.; Januszewski, S.; Czuczwar, S.J.; Andjus, P.R.; Pluta, R. Heterogeneity in brain distribution of activated microglia and astrocytes in a rat ischemic model of Alzheimer's disease after 2 years of survival. Aging 2020, 12, 12251-12267. [CrossRef] [PubMed]

2. Batista, C.R.A.; Gomes, G.F.; Candelario-Jalil, E.; Fiebich, B.L.; de Oliveira, A.C.P. Lipopolysaccharide-Induced Neuroinflammation as a Bridge to Understand Neurodegeneration. Int. J. Mol. Sci. 2019, 20, 2293. [CrossRef] [PubMed]

3. Bonow, R.H.; Aid, S.; Zhang, Y.; Becker, K.G.; Bosetti, F. The brain expression of genes involved in inflammatory response, the ribosome, and learning and memory is altered by centrally injected lipopolysaccharide in mice. Pharm. J. 2009, 9, 116-126. [CrossRef] [PubMed]

4. Mazin, P.V.; Jiang, X.; Fu, N.; Han, D.; Guo, M.; Gelfand, M.S.; Khaitovich, P. Conservation, evolution, and regulation of splicing during prefrontal cortex development in humans, chimpanzees, and macaques. RNA 2018, 24, 585-596. [CrossRef] 
5. Mazin, P.V.; Khaitovich, P.; Cardoso-Moreira, M.; Kaessmann, H. Alternative splicing during mammalian organ development. Nat. Genet. 2021, 53, 925-934. [CrossRef]

6. $\quad$ Pinner, E.; Gruper, Y.; Ben Zimra, M.; Kristt, D.; Laudon, M.; Naor, D.; Zisapel, N. CD44 Splice Variants as Potential Players in Alzheimer's Disease Pathology. J. Alzheimers Dis. 2017, 58, 1137-1149. [CrossRef] [PubMed]

7. Marchese, E.; Valentini, M.; Di Sante, G.; Cesari, E.; Adinolfi, A.; Corvino, V.; Ria, F.; Sette, C.; Geloso, M.C. Alternative splicing of neurexins $1-3$ is modulated by neuroinflammation in the prefrontal cortex of a murine model of multiple sclerosis. Exp. Neurol. 2021, 335, 113497. [CrossRef]

8. Makeyev, E.V.; Zhang, J.; Carrasco, M.A.; Maniatis, T. The MicroRNA miR-124 promotes neuronal differentiation by triggering brain-specific alternative pre-mRNA splicing. Mol Cell. 2007, 27, 435-448. [CrossRef]

9. Linares, A.J.; Lin, C.H.; Damianov, A.; Adams, K.L.; Novitch, B.G.; Black, D.L. The splicing regulator PTBP1 controls the activity of the transcription factor Pbx1 during neuronal differentiation. eLife 2015, 4, e09268. [CrossRef]

10. Vuong, J.K.; Lin, C.H.; Zhang, M.; Chen, L.; Black, D.L.; Zheng, S. PTBP1 and PTBP2 Serve Both Specific and Redundant Functions in Neuronal Pre-mRNA Splicing. Cell Rep. 2016, 17, 2766-2775. [CrossRef]

11. Georgilis, A.; Klotz, S.; Hanley, C.J.; Herranz, N.; Weirich, B.; Morancho, B.; Leote, A.C.; D'Artista, L.; Gallage, S.; Seehawer, M.; et al. PTBP1-Mediated Alternative Splicing Regulates the Inflammatory Secretome and the Pro-tumorigenic Effects of Senescent Cells. Cancer Cell. 2018, 34, 85.e9-102.e9. [CrossRef]

12. Pan, J.; Ma, N.; Yu, B.; Zhang, W.; Wan, J. Transcriptomic profiling of microglia and astrocytes throughout aging. J. Neuroinflamm. 2020, 17, 97. [CrossRef] [PubMed]

13. Geng, G.; Xu, C.; Peng, N.; Li, Y.; Liu, J.; Wu, J.; Liang, J.; Zhu, Y.; Shi, L. PTBP1 is necessary for dendritic cells to regulate T-cell homeostasis and antitumour immunity. Immunology 2021, 163, 74-85. [CrossRef] [PubMed]

14. Kim, J.H.; Jeong, K.; Li, J.; Murphy, J.M.; Vukadin, L.; Stone, J.K.; Richard, A.; Tran, J.; Gillespie, G.Y.; Flemington, E.K.; et al. SON drives oncogenic RNA splicing in glioblastoma by regulating PTBP1/PTBP2 switching and RBFOX2 activity. Nat. Commun. 2021, 12, 5551. [CrossRef] [PubMed]

15. Shishkina, G.T.; Gulyaeva, N.V.; Lanshakov, D.A.; Kalinina, T.S.; Onufriev, M.V.; Moiseeva, Y.V.; Sukhareva, E.V.; Babenko, V.N.; Dygalo, N.N. Identifying the Involvement of Pro-Inflammatory Signal in Hippocampal Gene Expression Changes after Experimental Ischemia: Transcriptome-Wide Analysis. Biomedicines 2021, 9, 1840. [CrossRef] [PubMed]

16. Ory, D.; Planas, A.; Dresselaers, T.; Gsell, W.; Postnov, A.; Celen, S.; Casteels, C.; Himmelreich, U.; Debyser, Z.; Van Laere, K.; et al PET imaging of TSPO in a rat model of local neuroinflammation induced by intracerebral injection of lipopolysaccharide. Nucl. Med. Biol. 2015, 42, 753-761. [CrossRef]

17. Bolger, A.M.; Lohse, M.; Usadel, B. Trimmomatic: A flexible trimmer for Illumina sequence data. Bioinformatics 2014, 30, 2114-2120. [CrossRef] [PubMed]

18. Dobin, A.; Gingeras, T.R. Mapping RNA-seq Reads with STAR. Curr. Protoc. Bioinform. 2015, 51, 11.14.11-11.14.19. [CrossRef]

19. Trapnell, C.; Williams, B.A.; Pertea, G.; Mortazavi, A.; Kwan, G.; van Baren, M.J.; Salzberg, S.L.; Wold, B.J.; Pachter, L. Transcript assembly and quantification by RNA-Seq reveals unannotated transcripts and isoform switching during cell differentiation. Nat. Biotechnol. 2010, 28, 511-515. [CrossRef] [PubMed]

20. Shen, S.; Park, J.W.; Lu, Z.X.; Lin, L.; Henry, M.D.; Wu, Y.N.; Zhou, Q.; Xing, Y. rMATS: Robust and flexible detection of differential alternative splicing from replicate RNA-Seq data. Proc. Natl. Acad. Sci. USA 2014, 111, E5593-E5601. [CrossRef]

21. Wang, G.; Oh, D.H.; Dassanayake, M. GOMCL: A toolkit to cluster, evaluate, and extract non-redundant associations of Gene Ontology-based functions. BMC Bioinform. 2020, 21, 139. [CrossRef]

22. Zhang, Y.; Chen, K.; Sloan, S.A.; Bennett, M.L.; Scholze, A.R.; O'Keeffe, S.; Phatnani, H.P.; Guarnieri, P.; Caneda, C.; Ruderisch, N.; et al. An RNA-sequencing transcriptome and splicing database of glia, neurons, and vascular cells of the cerebral cortex. J. Neurosci. 2014, 34, 11929-11947. [CrossRef]

23. Gueroussov, S.; Gonatopoulos-Pournatzis, T.; Irimia, M.; Raj, B.; Lin, Z.Y.; Gingras, A.C.; Blencowe, B.J. An alternative splicing event amplifies evolutionary differences between vertebrates. Science 2015, 349, 68-873. [CrossRef]

24. Ule, J.; Ule, A.; Spencer, J.; Williams, A.; Hu, J.S.; Cline, M.; Wang, H.; Clark, T.; Fraser, C.; Ruggiu, M.; et al. Nova regulates brain-specific splicing to shape the synapse. Nat. Genet. 2005, 37, 844-852. [CrossRef] [PubMed]

25. Ule, J.; Stefani, G.; Mele, A.; Ruggiu, M.; Wang, X.; Taneri, B.; Gaasterland, T.; Blencowe, B.J.; Darnell, R.B. An RNA map predicting Nova-dependent splicing regulation. Nature 2006, 444, 580-586. [CrossRef] [PubMed]

26. Porter, R.S.; Jaamour, F.; Iwase, S. Neuron-specific alternative splicing of transcriptional machineries: Implications for neurodevelopmental disorders. Mol. Cell Neurosci. 2018, 87, 35-45. [CrossRef] [PubMed]

27. Rawish, E.; Nording, H.; Münte, T.; Langer, H.F. Platelets as Mediators of Neuroinflammation and Thrombosis. Front. Immunol. 2020, 11, 548631. [CrossRef]

28. Jaffrey, S.R.; Wilkinson, M.F. Nonsense-mediated RNA decay in the brain: Emerging modulator of neural development and disease. Nat. Rev. Neurosci. 2018, 19, 715-728. [CrossRef] [PubMed]

29. Mironov, A.; Denisov, S.; Gress, A.; Kalinina, O.V.; Pervouchine, D.D. An extended catalogue of tandem alternative splice sites in human tissue transcriptomes. PLoS Comput. Biol. 2021, 7, e1008329. [CrossRef]

30. La Manno, G.; Soldatov, R.; Zeisel, A.; Braun, E.; Hochgerner, H.; Petukhov, V.; Lidschreiber, K.; Kastriti, M.E.; Lönnerberg, P.; Furlan, A.; et al. RNA velocity of single cells. Nature 2018, 560, 494-498. [CrossRef] [PubMed] 
31. Jumper, J.; Evans, R.; Pritzel, A.; Green, T.; Figurnov, M.; Ronneberger, O.; Tunyasuvunakool, K.; Bates, R.; Žídek, A.; Potapenko, A.; et al. Highly accurate protein structure prediction with AlphaFold. Nature 2021, 596, 583-589. [CrossRef]

32. Xue, Y.; Ouyang, K.; Huang, J.; Zhou, Y.; Ouyang, H.; Li, H.; Wang, G.; Wu, Q.; Wei, C.; Bi, Y.; et al. Direct conversion of fibroblasts to neurons by reprogramming PTB-regulated microRNA circuits. Cell 2013, 152, 82-96. [CrossRef]

33. Qian, H.; Kang, X.; Hu, J.; Zhang, D.; Liang, Z.; Meng, F.; Zhang, X.; Xue, Y.; Maimon, R.; Dowdy, S.F.; et al. Reversing a model of Parkinson's disease with in situ converted nigral neurons. Nature 2020, 582, 550-556. [CrossRef] [PubMed]

34. Xue, Y.; Qian, H.; Hu, J.; Zhou, B.; Zhou, Y.; Hu, X.; Karakhanyan, A.; Pang, Z.; Fu, X.D. Sequential regulatory loops as key gatekeepers for neuronal reprogramming in human cells. Nat. Neurosci. 2016, 19, 807-815. [CrossRef] [PubMed]

35. Wang, X.; Li, Y.; Fan, Y.; Yu, X.; Mao, X.; Jin, F. PTBP1 promotes the growth of breast cancer cells through the PTEN/Akt pathway and autophagy. J. Cell Physiol. 2018, 233, 8930-8939. [CrossRef] [PubMed]

36. Reshetnikov, V.V.; Kisaretova, P.E.; Ershov, N.I.; Shulyupova, A.S.; Oshchepkov, D.Y.; Klimova, N.V.; Ivanchihina, A.V.; Merkulova, T.I.; Bondar, N.P. Genes associated with cognitive performance in the Morris water maze: An RNA-seq study. Sci Rep. 2020, 10, 22078. [CrossRef] [PubMed]

37. Sheng, Z.; Sun, Y.; Zhu, R.; Jiao, N.; Tang, K.; Cao, Z.; Ma, C. Functional Cross-Talking between Differentially Expressed and Alternatively Spliced Genes in Human Liver Cancer Cells Treated with Berberine. PLoS ONE 2015, 10, e0143742. [CrossRef]

38. Udy, D.B.; Bradley, R.K. Nonsense-mediated mRNA decay uses complementary mechanisms to suppress mRNA and protein accumulation. Life Sci. Alliance 2021, 5, e202101217. [CrossRef] 